\title{
FOLIICOLOUS FUNGI OF ANDAMAN ISLANDS, INDIA
}

\section{V.B. Hosagoudar ${ }^{1}$, Sam P. Mathew ${ }^{2}$ \& Divya Babu ${ }^{3}$}

1,2,3 Jawaharlal Nehru Tropical Botanic Garden and Research institute, Palode, Thiruvananthapuram, Kerala 695562, India

11vbhosagoudar@rediffmail.com (corresponding author), ${ }^{2}$ sampmatthew@rediffmail.com, ${ }^{3}$ divyababuob@gmail.com

ISSN

Online 0974-7907 Print 0974-7893

OPEN ACCESS

Abstract: One month field collection tour resulted in the collection of more than 500 foliicolous fungi. Of these, 152 collections have been identified, resulted in recording 60 fungal taxa. Of these, one new genus (Andamanomyces), 16 new species (Andamanomyces fragariae, Asterediella anaxagoreae, Asteridiella colocasiae, Asterina eleocarpigena, Asterostomella dhanikariensis, Asterostomella farrargunjensis, Irenopsis andamanica, Meliola andamanica, Meliola canarifolia, Meliola chukrasiicola, Meliola harrietensis, Meliola myristicacearam, Meliola parishiae, Meliola savarkarii, Meliola ternstroemiicola and Schiffnerula chukrasiae) and one new variety (Meliola pithecellobii var. indica) are discovered. Meliola palmicola is reported here for the first time from India. All these taxa are listed but the novel taxa are described and illustrated in detail.

Keywords: Andaman Islands, foliicolous fungi, India, new species.

DOI: http://dx.doi.org/10.11609/JoTT.o3652.5447-63

Editor: H.C. Nagaveni, Retd. Scientist, Institute of Wood Science and Technology, Bengaluru, India.

Date of publication: 26 February 2014 (online \& print)

Manuscript details: Ms \# 03652 | Received 03 August 2013 | Final received 07 December 2013 | Finally accepted 31 December 2013

Citation: Hosagoudar, V.B., S.P. Mathew \& D. Babu (2014). Foliicolous fungi of Andaman Islands, India. Journal of Threatened Taxa 6(2): 5447-5463; http://dx.doi. org/10.11609/JoTT.03652.5447-63

Copyright: @ Hosagoudar et al. 2014. Creative Commons Attribution 3.0 Unported License. JoTT allows unrestricted use of this article in any medium, reproduction and distribution by providing adequate credit to the authors and the source of publication.

Funding: This project is sponsored by the Department of Science and Technology, New Delhi.

Competing Interest: The authors declare no competing interests.

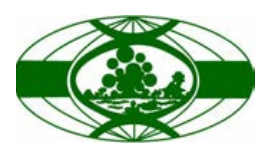

Author Details and Contribution: V.B. HOSAGOUDAR is the PI of this project and has identified the fungi; SAM P. MATHEW has identified the host plants and the DIVYA BABU is research scholar.

Acknowledgements: We gratefully acknowledge Dr. Paramjit Singh, Director, Botanical Survey of India, Kolkata; Dr. C. Murugan, Head, Dr. T.A.M. Jagadeesh Ram and Dr. Lalji Singh, Senior Scientists, Botanical Survey of India, Andaman and Nicobar Circle, Port Blair, for their guidance and help in various ways during the field collection tour to Andaman Islands. Forest officials were generous in permitting us to collect the infected leaves for the research. Dr. P.G. Latha, Director, JNTBGRI is thanked for the facilities. 


\section{INTRODUCTION}

Andaman Islands, the pristine Indian archipelago in the Bay of Bengal is about 647 nautical miles away from the Coromandel coast of the Peninsular India, located between $92^{\circ} 12^{\prime \prime}-93^{\circ} 17^{\prime \prime} \mathrm{E} \& 6^{\circ} 45^{\prime \prime}-13^{\circ} 45^{\prime \prime} \mathrm{N}$, comprise about 325 islands, islets and rocks which cover an area of $6,408 \mathrm{~km}^{2}$ (Image 1). Geologically, these islands are considered as the emergent peaks of a submerged mountain range in continuation with the ArakanYoma Mountains of the Myanmar. The entire length of Andaman group of islands is estimated to be $467 \mathrm{~km}$ while the maximum width is about $51.5 \mathrm{~km}$. Landfall Island demarcates the northern end of the Andaman Archipelago while Little Andaman Island occupies the extreme southern end of this group. The two volcanic islands, viz., Narcondom Island and Barren Island, demarcate the eastern boundary while Interview Island and Sentinel Islands delimit the western extremity. The Andaman Archipelago consists of four major groups of islands such as North Andamans, Middle Andamans, South Andamans and the Little Andaman. The former three groups are collectively known as Great Andamans. The biggest island in this group is South Andaman (2,021 $\mathrm{km}^{2}$ ) and smallest is the Aves Island $\left(0.2 \mathrm{~km}^{2}\right)$.

The toponymy of Andaman Islands is in different names since $2^{\text {nd }}$ century. Ptolomy, the famous Roman geographer referred to these islands as 'Agmate' or 'Aginae' in his travelogues. It has been mentioned as 'Timattive' in 'Tanjor Incriptions' of 1050AD. The Malayan people who came to these islands for capturing slaves referred these islands as 'Handuman'. It appears that the present name has originated from 'Hanuman', the monkey god of the Indian epic, Ramayana. These islands are also known as Bay Islands, since in the Bay of Bengal. 'Kala Pani' is another name, perhaps given by the Indian freedom fighters during the Indian freedom struggle. This name is derived from the Sanskrit word 'Kal-Pani' which means the water of death or place of banishment but not the 'black water' as mentioned in many literature. Whatever be the different names in the remote past, these islands are referred to the name given by the Malayans with slight phonetic changes seems to be more logic in toponymy of these islands.

The proximity of these islands towards equator contributes tropical climate with an average temperature of $30^{\circ} \mathrm{C}$, rainfall of $3100 \mathrm{~mm}$ and relative humidity of $77 \%$, facilitate luxuriant growth of rainforests. According to an official estimation, around $83 \%$ of the land area of these islands are covered by dense lowland rain forests which could be designated as 'humid tropics' ranging from sea level to an altitude up to $732 \mathrm{~m}$ at Saddle Peak (North Andamans). From phytogeographical point of view, the insular flora of the Andaman and Nicobar Islands is remarkable as the transitional zone vegetation between the South and Southeast Asia as well as the only stronghold of Malesian floristic elements within the Indian Territory. The geographical isolation of these islands from the major land masses of South and Southeast Asia over millions of years have resulted in unique endemism and coupled with multi-dimensional floristic affinities towards nearer and distant geographical regions such as northeastern India, Southeast Asia, Malesia and to the Western Ghats of the peninsular India. According to a recent official estimation carried out by Pandey \& Diwakar from the Botanical Survey of India in 2008, the Bay Islands are known to host about 2,428 Angiosperm taxa including 294 endemics.

The general topography of the islands is very irregular and undulating. The irregular deeply indented coastal lines result in many creeks and coves. The highest point in the Bay of Bengal is the 'Saddle Peak' $(732 \mathrm{~m})$ in North Andamans. The other major heights of Andaman archipelago are Mount Diavolo (505m), Mount Oldham (304m), Mount Angelica (458m) of the Middle Andamans, Mount Ford $(428 \mathrm{~m})$ of the Rutland Island (South Andamans), Mount Harriet ranges of the South Andamans which reaches the maximum height of $460 \mathrm{~m}$ at Mount Koyob of the same ranges. The other major heights are the Mount Hext (424m), Mount Warden (422m), Mount Harriet (398m), Mount Goodridge $(321 \mathrm{~m})$, Mount Carpenter $(310 \mathrm{~m})$ and the Mount Nappier (304m)

The insular types of vegetation ranging from shallow marine ecosystems to the inland forest ecosystems could be broadly classified into two different zonal types such as coastal or littoral vegetation and the inland vegetation, based on the proximity of the sea. The zonal types have been further subdivided into several types based on the floristic composition owing to the influence of other edaphic, climatological and physical factors such as humidity, soil types, daily tides, retentivity of soil, altitude, etc. The coastal or littoral forest vegetation constitutes marine aquatic vegetation, mangrove forest vegetation, tidal swamp vegetation and littoral forest vegetation along the sea coasts. The inland vegetation comprises the giant evergreen forests, Andaman tropical evergreen forests, southern hill top tropical evergreen forests, semi evergreen forests, moist deciduous forests, grasslands, inland aquatic vegetation and manmade vegetations.

However, in such an interesting floristically rich 


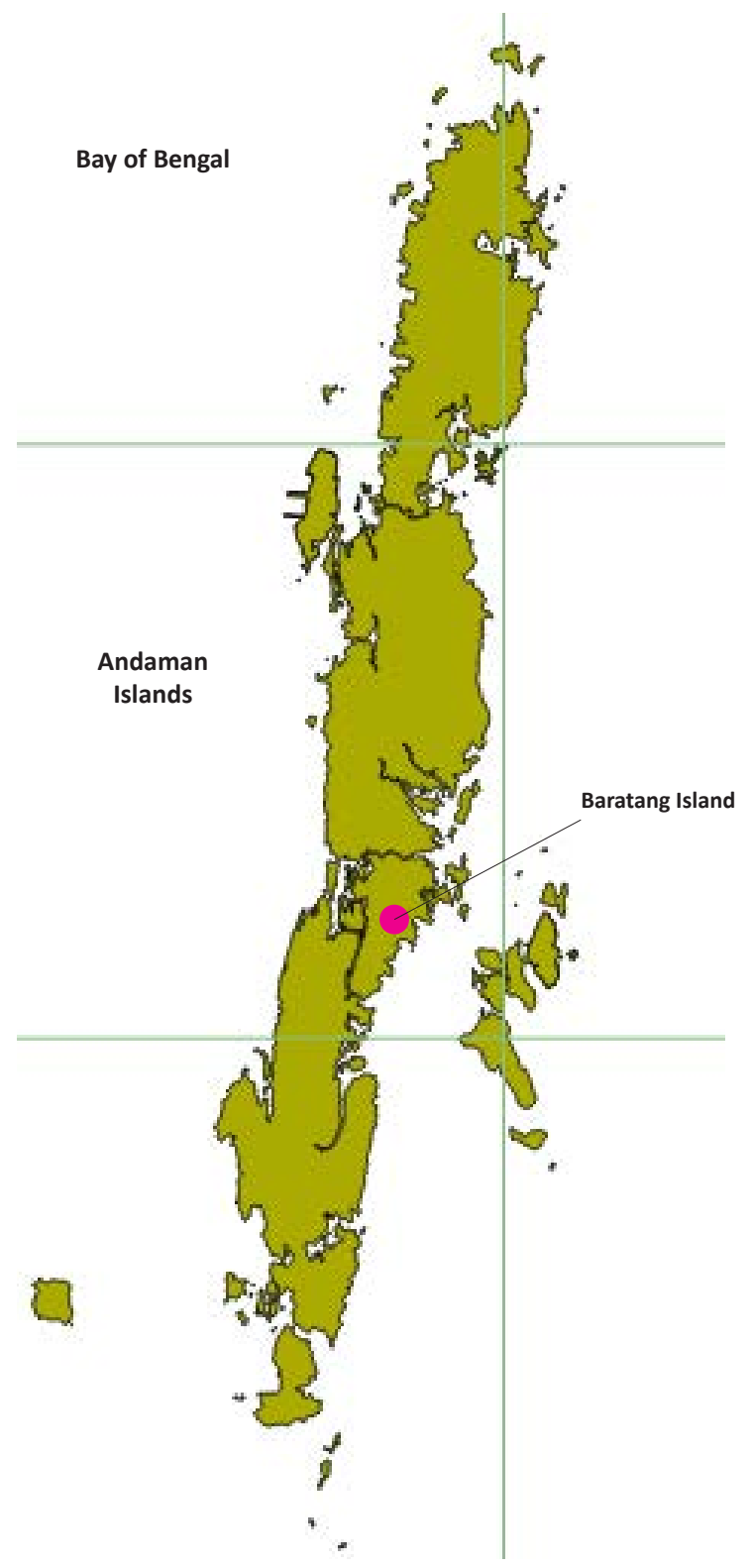

Image 1. Andaman Islands

forests, the study of lower groups is very much meager. In case of foliicolous fungi alone, generally it is stated that there will be 10 fungi to each plant species. This archipelago has 2,428 plant species, it is expected more than 24,000 fungi against the just known 10 foliicolous fungal species (Hosagoudar \& Mathew 2000).

\section{LIST OF FUNGAL TAXA}

Acroconidiellina arecae (Berk. \& Br.) M.B. Ellis

Materials examined: TBGT 6528, PBL 121, 16.xii.2012, on leaves of Areca catechu (Arecaceae), Billiground, Mayabandar, North Andaman, coll. V.B. Hosagoudar et al.

\section{Andamanomyces gen. nov. V.B. Hosagoudar \\ (Asterinales, Lembosiaceae) \\ urn:Isid:indexfungorum.org:names:807674}

Fungus foliicolous, ectophytic. Mycelium ectophytic, brown, septate, branched, appressoriate. Appressoria solitary but mostly in couplets like Gangamyces, unicellular, lumen between both the appressoria is continuous or separated by cell wall of the mycelial cells. Thyriothecia oval, ellipsoidal with central vertical suture formed by dissolving or splitting; asci globose, 8-spored, bitunicate; ascospores brown, conglobate, oblong, uniseptate, constricted at the septum.

Etymology: Fungus named after the Andaman Islands.

Type: Andamanomyces fragariae sp. nov.

This genus is similar to Gangamyces but differs from it in having elliptical thyriothecia with central vertical suture as is in the case of Asterolibertia and Cirsosia.

\section{Andamanomyces fragariae sp. nov.}

(Fig. 1) urn:Isid:indexfungorum.org:names: 807684

Materials examined: TBGT 6666 (holotype), PBL 259 (isotype); TBGT 6670, PBL 263; TBGT 6675, PBL 269, 11.xii.2012, on leaves of Fragaria sp. (Rosaceae), Mount Harriet National Park, coll. Hosagoudar et al.

Colonies epiphyllous, dense, up to $3 \mathrm{~mm}$ in diameter. Hyphae straight to substraight, branching opposite to irregular at acute angles, closely to loosely reticulate, cells 35-45x5-7 $\mu \mathrm{m}$. Appressoria in opposite couplet, rarely solitary forming stellate lumen in the cells on which they are born, scattered to closely scattered, ovate, globose, margin entire to sublobate, couplet horizontal, 22-27 $\mu \mathrm{m}$ broad, appressoria individually 8-10 $\mu \mathrm{m}$ broad, lumen quadrangular in shape, often a link of mycelial cell is visible, with its points towards upper and lower septa and the other two directed towards the centre of lumen of appressoria, 10-15x5$7 \mu \mathrm{m}$. Thyriothecia oval, ellipsoidal to elongated, scattered to loosely grouped, longitudinally dehisced

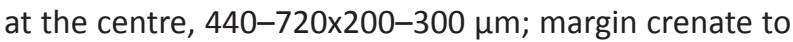
fimbriate, fringed hyphae flexuous; asci oval to globose, 
octosporous, $32-34 \times 17-20 \mu \mathrm{m}$; ascospores oblong to cylindrical, conglobate, 1-septate, constricted at the

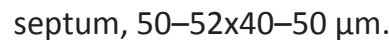

This genus differs from Gangamyces in having elliptical thyriothecia with vertical suture (Hosagoudar 2012).

Etymology: Named after its host plant.

\section{Asteridiella anamalaiana Hosag}

Materials examined: TBGT 6418, PBL 11, 17.xii.2012, on leaves of Sterculia sp. (Sterculiaceae), South creek, Baratang, Middle Andaman, coll. V.B. Hosagoudar et al.; TBGT 6508, PBL101, 15.xii.2012, Kallara junction, Diglipur, North Andaman, coll. V.B. Hosagoudar et al.; TBGT 6486, PBL 079, 12.xii.2012, Dhanikari, South Andaman, coll. V.B. Hosagoudar et al.; TBGT 6448, PBL 41, 20.xii.2012, Farrar gunj, South Andaman, V.B. Hosagoudar et al.; TBGT 6451, PBL 44, 21.xii.2012, Tushnabad, South Andaman, coll. V.B. Hosagoudar et al.

\section{Asteridiella anaxagoreae sp nov.}

(Fig. 2) urn:Isid:indexfungorum.org:names: 807685

Materials examined: TBGT 6680 (holotype), PBL 274 (isotype), 8.xii.2012, on leaves of Anaxagorea javanica (Annonaceae), Sholbay, South Andaman, V.B. Hosagoudar et al.

Colonies epiphyllous, subdense, up to $4 \mathrm{~mm}$ in diameter. Hyphae straight to substraight, branching mostly opposite to rarely alternate at acute to wide angles, loosely to closely reticulate, cells $25-30 \times 5-7$ $\mu \mathrm{m}$. Appressoria alternate to unilateral, straight to slightly curved, antrorse to sub antrorse, 20-25 $\mu \mathrm{m}$ long; head cells globose, entire, clavate, truncate, angular, 15-20x10-15 $\mu \mathrm{m}$. Phialides born on a separate mycelial branch, opposite to alternate, ampulliform, 17-22×6-7 $\mu \mathrm{m}$. Perithecia scattered, up to $140 \mu \mathrm{m}$ in diameter; perithecial wall cells conoid to ampulliform, up to $15 \mu \mathrm{m}$ long; ascospores oblong to cylindrical, 4-septate, constricted at the septa, 32-37x12-17 $\mu \mathrm{m}$.

Asteridiella subglabroides Hansf. is known on Annonaceae member from British Guiana (Hansford 1961) but Asteridiella anaxagoreae differs from it in having mostly entire head cells of appressoria and longer ascospores.

Etymology: Named after its host plant.

\section{Asteridiella colocasiae sp. nov.}

(Fig. 3 \& Image 1)

urn:Isid:indexfungorum.org:names: 807686

Materials examined: TBGT 6677 (holotype), PBL 271 (isotype), 8.xii.2012, on leaves of Colocasia sp. (Aracaceae), Wright Myo, South Andaman, coll. V.B. Hosagoudar et al.

Colonies epiphyllous, dense, scattered, up to $1 \mathrm{~mm}$

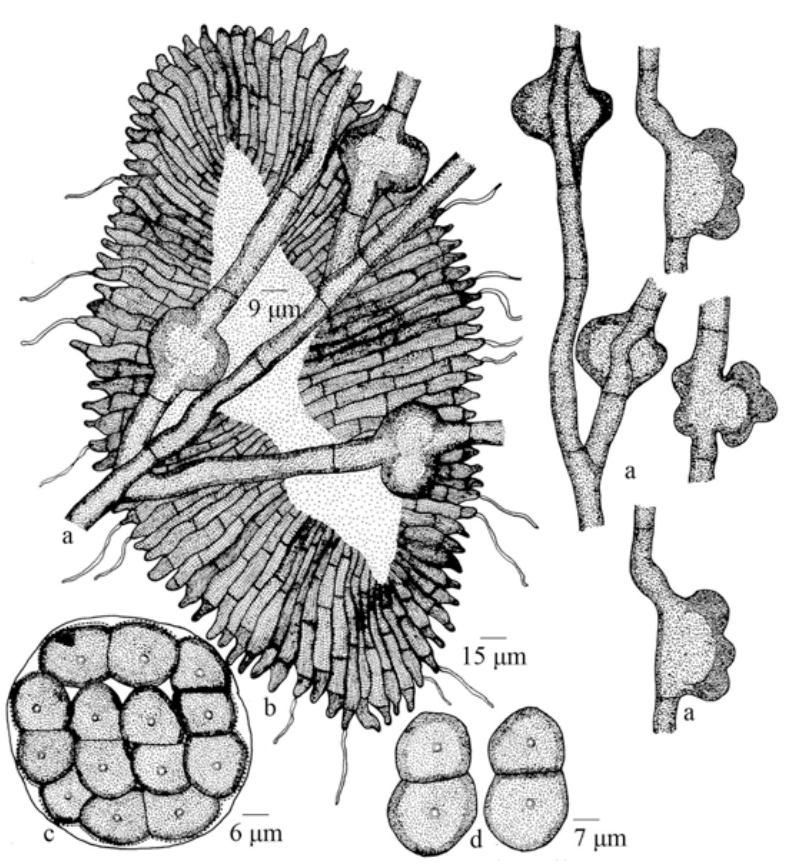

Figure 1. Andamanomyces fragariae sp. nov.

a - Appressoriate mycelium; b - Thyriothecium; c - Ascus; d - Ascospores

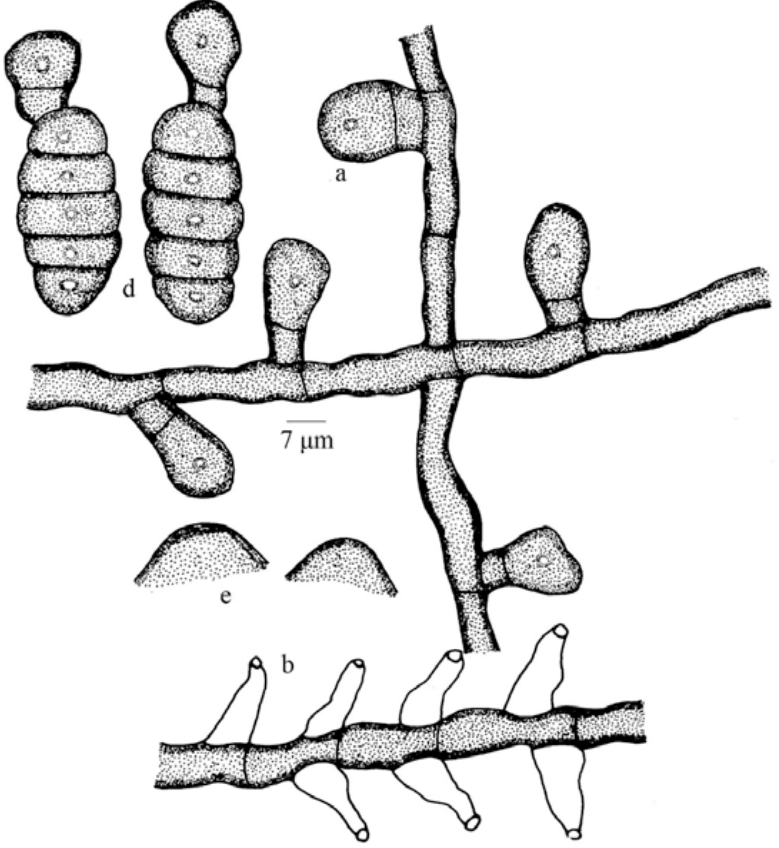

Figure 2. Asteridiella anaxagoreae sp. nov.

a - Appressorium; b - Phialide; d - Ascospores; e - Peridial cells 


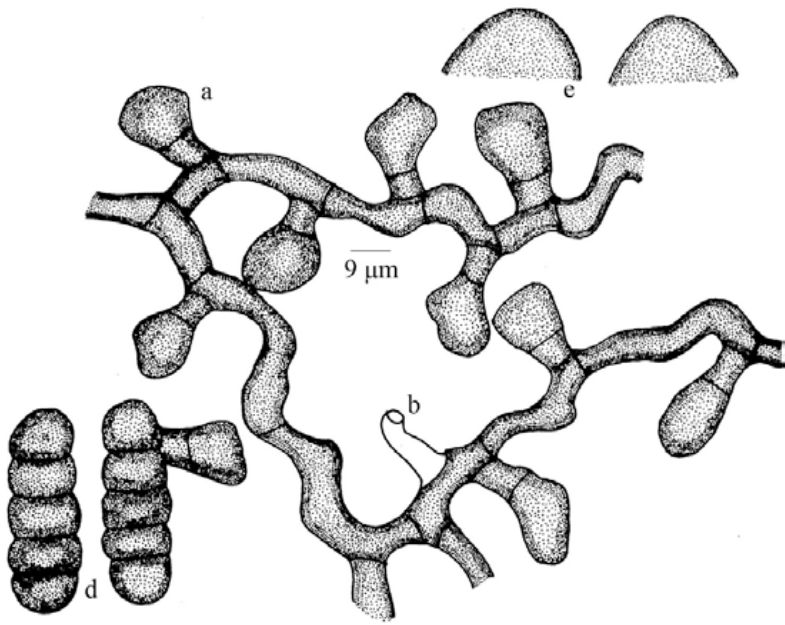

Figure 3. Asteridiella colocasiae sp. nov.

a - Appressorium; b - Phialide; $d$ - Ascospores; e - Peridial cells
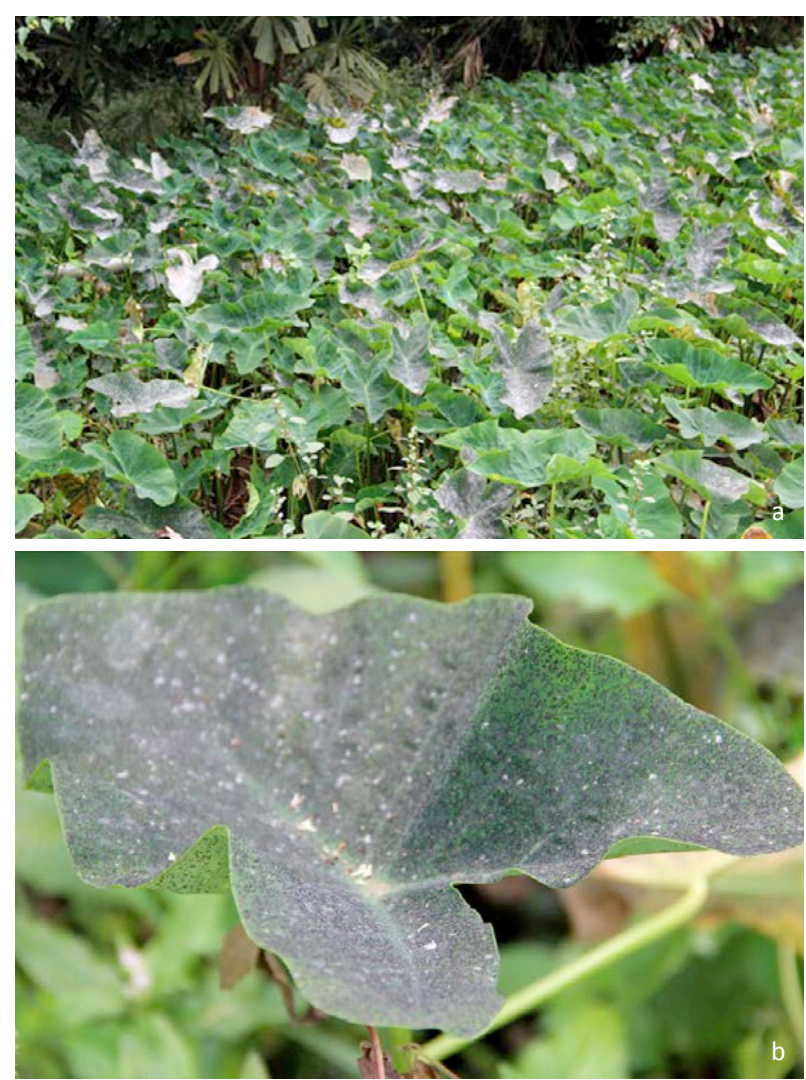

Image 1. a - Infected Colocasia vegetation; b - Asteridiella colocasiae sp. nov. on Colacasia sp.

in diameter, confluent. Hyphae flexuous to crooked, branching alternate to irregular at acute to wide angles, form a loose mycelial net, cells $15-22 \times 6-7 \mu \mathrm{m}$. Appressoria alternate to unilateral, antrorse, subantrorse to spreading, straight to curved, 20-25 $\mu \mathrm{m}$ long; stalk cells cylindrical to cuneate, 5-10 $\mu \mathrm{m}$ long; head cells straight to curved, ovate, clavate, globose, truncate at the apex, angular, entire, $12-15 \times 10-15 \mu \mathrm{m}$. Phialides mixed with appressoria, ampulliform, 15-20x5-7 $\mu \mathrm{m}$. Perithecia scattered to loosely grouped, up to $180 \mu \mathrm{m}$ in diameter; perithecial wall cells mammiform, up to $12 \mu \mathrm{m}$ long; ascospores ellipsoidal, 4-septate, 42-45×12-14 $\mu \mathrm{m}$, mostly constricted at the septa.

Asteridiella pothodis (Hansf. \& Thirm.) Hansf. is known on Pothos scandens from the Western Ghats of Peninsular India (Hansford \& Thirumalachar 1948; Hansford 1961). Asteridiella colocasiae differs from it in loosely forming hyphal net.

Etymology: Named after its host plant.

Asteridiella combreti (Stev.) Hansf. var. leonensis Hansf.

Materials examined: TBGT 6513, PBL 106, 14.xii.2012, on leaves of Terminalia bialata (Combretaceae), Lakshmipur Nursery, North Andaman, coll. V.B. Hosagoudar et al.

\section{Asterina claviflori Kar \& Maity}

Materials examined: TBGT 6408, PBL 001, 17.xii.2012, on leaves of Syzygium sp. (Myrtaceae), Mangrove forest guest house, Middle Andaman, coll. V.B. Hosagoudar et al.; TBGT 6470, PBL 063, 14.xii.2012, Millengram, Diglipur, North Andaman, coll. V.B. Hosagoudar et al.

\section{Asterina diplocarpa Cooke}

Materials examined: TBGT 6483, PBL 076, 20.xii.2012, on leaves of Sida sp. (Malvaceae), Farrar gunj, South Andaman, coll. V.B. Hosagoudar et al.; TBGT 6523, PBL 116, 14.xii.2012, Shibpur, Diglipur, North Andaman, coll. V.B. Hosagoudar et al.

\section{Asterina elaegni (Sydow) Sydow \& Petrak}

Materials examined: TBGT 6489, PBL 082, 16.xii.2012, on leaves of Elaeagnus sp. (Elaeagnaceae), Kallara junction, Diglipur, North Andaman, coll. V.B. Hosagoudar et al.

\section{Asterina eleocarpigena sp. nov.}

(Fig. 4) urn:Isid:indexfungorum.org:names: 807687

Materials examined: TBGT 6676 (holotype) PBL 270 (isotype), 8.xii.2012, on leaves of Elaeocarpus sp. (Elaeocarpaceae), South Andaman, coll. V.B. Hosagoudar et al.

Colonies epiphyllous, thick, scattered, up to $6 \mathrm{~mm}$ in diameter, confluent. Hyphae mostly straight to substraight, branching opposite to irregular at acute to wide angles, loosely to closely reticulate, cells $17-25 \times 5-$ 


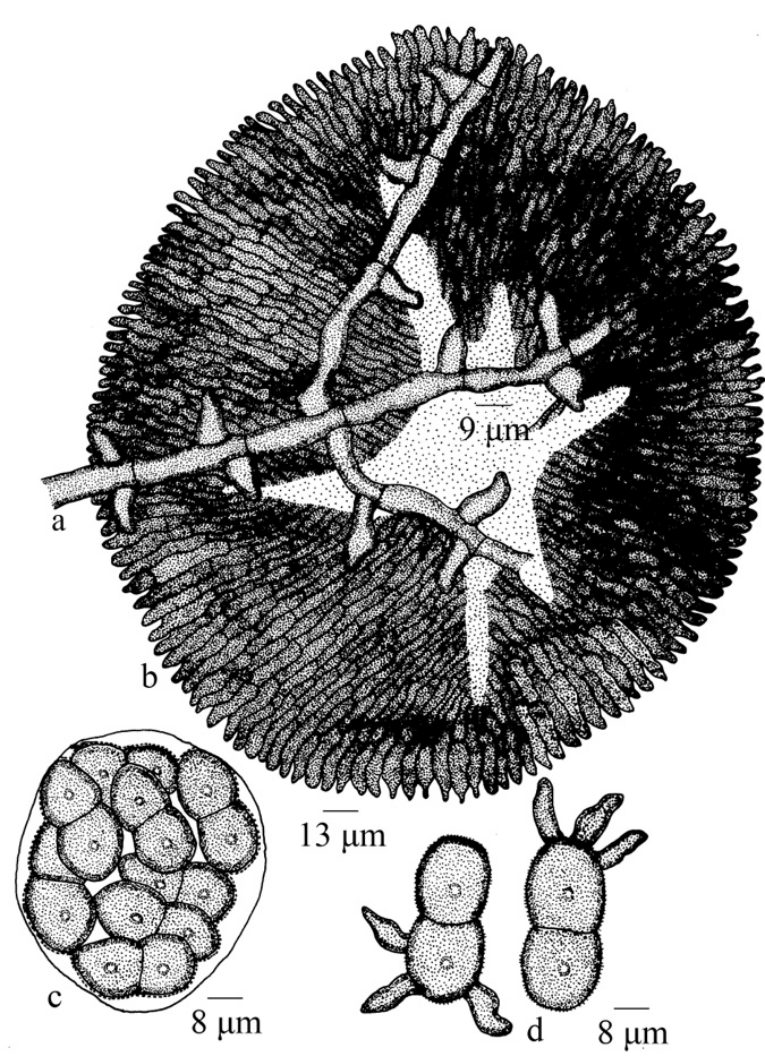

Figure 4. Asterina eleocarpigena sp. nov.

a.Appressoriate mycelium, b. Thyriothecium, c. Ascus, d. Ascospores

$6 \mu \mathrm{m}$. Appressoria opposite (90\%) to alternate, straight to curved, antrorse, subantrorse to retrorse, ovate, often ampulliform, attenuated and broadly rounded towards apex, 12-15x5-7 $\mu \mathrm{m}$. Thyriothecia scattered, rarely one to two connate, up to $270 \mu \mathrm{m}$ in diameter, stellately dehisced at the centre, margin crenate to fimbriate, fringed hyphae flexuous, devoid of appressoria; asci globose, octosporous, 47-62×45-60 $\mu \mathrm{m}$ in diameter; ascospores oblong, conglobate, brown, uniseptate, slightly constricted at the septum, 35-37×15-17 $\mu \mathrm{m}$, wall tuberculate.

There are six Asterina species, namely, Asterina borneensis Hansf., Asterina elaeocarpi Sydow, Asterina elaeocarpi var. ovalis Kar \& Ghosh, Asterina eleocarpicola Hansf., Asterina elaeocarpi-kobanmochi Yamam. and Asterina gamsii are known on this host genus. However, Asterina elaeocarpigena differs from all in having $90 \%$ opposite, ovate, conoid to ampulliform appressoria (Hosagoudar \& Abraham 2000; Hosagoudar 2009, 2012).

Etymology: Named after its host plant.

\section{Asterina escharoides Sydow}

Materials examined: TBGT 6493, PBL 086, 15.xii.2012, on leaves of Quisqualis indica (Combretaceae), Kalighat,
Diglipur, North Andaman, coll. V.B. Hosagoudar et al.; TBGT 6531, PBL 124, 14.xii.2012, Radhanagar, Diglipur, North Andaman, coll. V.B. Hosagoudar et al.; TBGT 6525, PBL 118, 14.xii.2012, Shibpur, Diglipur, North Andaman, coll. V.B. Hosagoudar et al.; TBGT 6510, PBL 103, 17.xii.2012, Krishnanagar, Baratang, Middle Andaman, coll. V.B. Hosagoudar et al.

\section{Asterina jambolana Kar \& Maity}

Materials examined: TBGT 6434, PBL 027, 15.xii.2012, on leaves of Syzygium sp. (Myrtaceae), Central forest nursery, Nabagram, Diglipur, North Andaman, coll. V.B. Hosagoudar et al.; TBGT 6437, PBL 030, 15.xii.2012, Keralapuram, Diglipur, North Andaman, coll. V.B. Hosagoudar et al.; TBGT 6516, PBL109, 6.xii.2012, North Bay, South Andaman, coll. V.B. Hosagoudar et al.; TBGT 6458, PBL 051, 20.xii.2012, Farrar gunj, South Andaman, coll. V.B. Hosagoudar et al.; TBGT 6522, PBL 115, 6.xii.2012, North Bay, South Andaman, coll. V.B. Hosagoudar et al.; TBGT 6520, PBL 113, 6.xii.2012, Syzygium cumini, North Bay, South Andaman, coll. V.B. Hosagoudar et al.

\section{Asterina lawsoniae Nymn.}

Materials examined: TBGT 6410, PBL 003, 17.xii.2012, on leaves of Lawsonia inermis (Lythraceae), Krishna nagar, Baratang, Middle Andaman, coll. V.B. Hosagoudar et al.; TBGT 6521, PBL 114, 14.xii.2012, Millengram, Diglipur, North Andaman, coll. V.B. Hosagoudar et al.; TBGT 6497, PBL 090, 11.xii.2012, Bamboo flat, South Andaman, coll. V.B. Hosagoudar et al.; TBGT 6490, PBL 083, 15.xii.2012, Govt. Secondary school, Ramnagar, North Andaman, coll. V.B. Hosagoudar et al.

\section{Asterina triumfetticola Yamam.}

Materials examined: TBGT 6420, PBL 013, 17.xii.2012, on leaves of Triumfetta sp. (Tiliaceae), Krishna nagar, Baratang, Middle Andaman, coll. V.B. Hosagoudar et al.; TBGT 6524, PBL 117, 14.xii.2012, Shibpur, Diglipur, North Andaman, coll. V.B. Hosagoudar et al.

\section{Asterostomella dhanikariensis sp. nov.}

(Fig. 5) urn:Isid:indexfungorum.org:names: 807688

Materials examined: TBGT 6671 (holotype), PBL 264 (isotype), 7.xii.2012, on leaves of Tetrastigma sp. (Vitaceae), Dhanikari, Vandoor, South Andaman, coll. V.B. Hosagoudar et al.

Colonies hypophyllous, subdense, up to $2 \mathrm{~mm}$ in diameter. Hyphae flexuous to crooked, branching irregular at acute to wide angles, loosely to closely 


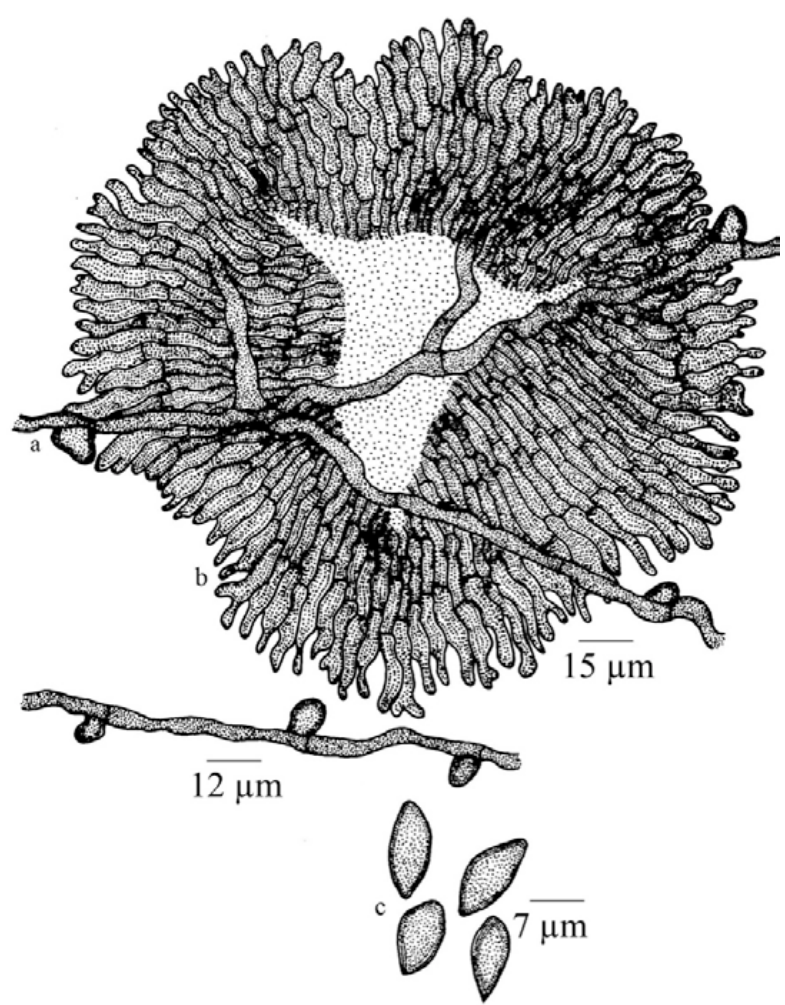

Figure 5. Asterostomella dhanikariensis sp. nov.

a - Appressoriate mycelium; b - Pycnothyrium; c - Pycnothyriospores

reticulate, cells $15-22 \times 2-3 \mu \mathrm{m}$. Appressoria distantly placed, alternate to unilateral, straight to curved, unicellular, ovate, globose, mammate, often slightly stipulate, entire, angular, sublobate, 5-10x5-7 $\mu \mathrm{m}$. Pycnothyria numerous, densely scattered, orbicular, up to $220 \mu \mathrm{m}$ in diameter, stellately dehisced at the centre. Pycnothyriospores pale to deep brown, oval, pyriform, fusiform, often with a hyaline band at the centre, 1015x5-7 $\mu \mathrm{m}$.

This species differs from other known Asterina species on the members of the family Vitaceae in having fusiform pycnothyriospores and distantly placed, mammiform, subsessile to stipulate appressoria (Hosagoudar \& Abraham 2000; Hosagoudar 2012).

Etymology: Named after its host plant.

\section{Asterostomella farrargunjensis sp. nov.}

(Fig. 6) urn:Isid:indexfungorum.org:names: 807689

Materials examined: TBGT 6669 (holotype), PBL 262 (isotype); TBGT 6673, PBL 267, 20.xii.2012, on leaves of Tetracera sermentosa var. indica (Dilleniaceae), Farrar gunj, South Andaman, coll. V.B. Hosagoudar et al.

Colonies hypophyllous, subdense, up to $2 \mathrm{~mm}$ in diameter, confluent. Hyphae flexuous to crooked, branching irregular at acute angles, loosely to closely reticulate, cells 22-27x3-5 $\mu \mathrm{m}$. Appressoria few, distantly placed, unicellular, oblong, ovate, entire, 6-7x5-7 $\mu \mathrm{m}$. Pycnothyria closely scattered, orbicular, up to $180 \mu \mathrm{m}$ in diameter, margin crenate, dissolved and widely opened at the centre; pycnothyriospores ellipsoidal, pyriform, 12-17x7-10 $\mu \mathrm{m}$, wall smooth.

This species is similar to Asterostomella stipitipodia Farr. in having the similar morphology with the appressoria (Farr 1987). However differs from it in having smaller pcynothyriospores (12-17x7-10 vs $18-$ $28 \times 10-16 \mu \mathrm{m})$.

Etymology: Named after its collection locality.

\section{Chaetomium sp.}

Materials examined: TBGT 6514, PBL 107, 16.xii.2012, on leaves of Hyptis sp. (Lamiaceae), Bakulthala, Middle Andaman, V.B. Hosagoudar et al.

\section{Cirsosia arecacearum Hosag. \& Pillai}

Materials examined: TBGT 6428, PBL 021, 17.xii.2012, on leaves of Calamus sp. (Arecaceae), Jarwa creek, Baratang, Middle Andaman, coll. V.B. Hosagoudar et al.; TBGT 6452, PBL 045, 21.xii.2012, Tushnabad, South Andaman, coll. V.B. Hosagoudar et al.; TBGT 6496, PBL 089, 18.xii.2012, Sholbay 14, South Andaman, coll. V.B. Hosagoudar et al.; TBGT 6482, PBL 075, 12.xii.2012, Dhanikari, South Andaman, coll. V.B. Hosagoudar et al.; TBGT 6480, PBL 073, 12.xii.2012, Dhanikari, South Andaman, coll. V.B. Hosagoudar et al.; TBGT 6476, PBL 069, 14.xii.2012, Radhanagar, Diglipur, North Andaman, coll. V.B. Hosagoudar et al.; TBGT 6472, PBL 065, 14.xii.2012, Shipur, Diglipur, North Andaman, coll. V.B. Hosagoudar et al.; TBGT 6460, PBL 053, 20.xii.2012, Farrar gunj check post, South Andaman, coll. V.B. Hosagoudar et al.; TBGT 6447, PBL 040, 20.xii.2012, Farrar gunj, South Andaman, coll. V.B. Hosagoudar et al.; TBGT 6445, PBL 038 21.xii.2012, Tushnabad, South Andaman, coll. V.B. Hosagoudar et al.

\section{Irenopsis andamanica sp. nov.}

(Fig. 7) urn:Isid:indexfungorum.org:names: 807690

Materials examined: TBGT 6682 (holotype), PBL 276 (isotype), 8.xii.2012, on leaves of Tabernaemontana sp. (Apocynaceae), Sholbay, South Andaman, coll. V.B. Hosagoudar et al.

Colonies epiphyllous, dense, up to $6 \mathrm{~mm}$ in diameter. Hyphae straight to flexuous, branching opposite to irregular at acute to wide angles, loosely to closely reticulate, cells $20-25 \times 6-7 \mu \mathrm{m}$. Appressoria alternate 


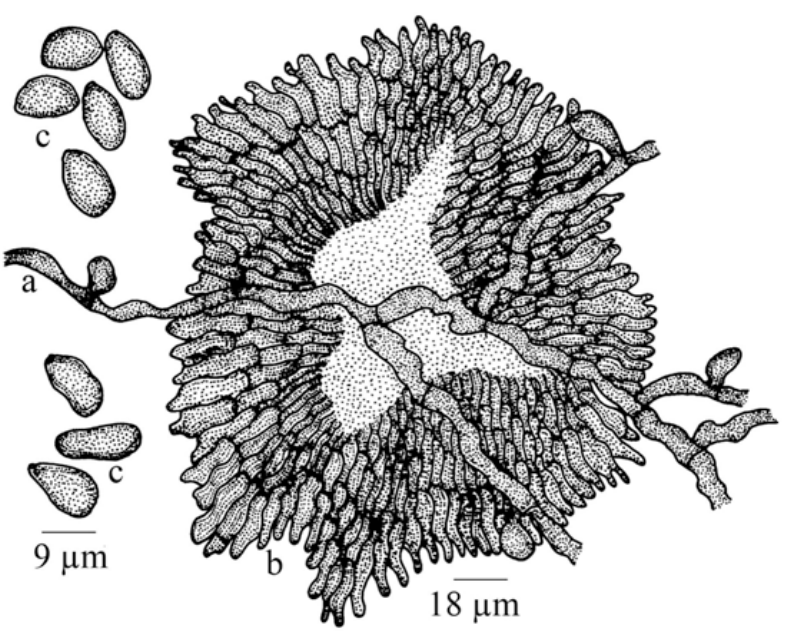

Figure 6. Asterostomella farrargunjensis sp. nov.

a - Appressoriate mycelium; b - Pycnothyrium; c - Pycnothyriospores

to unilateral, straight to curved, antrorse, sub antrorse to retrorse, 20-27 $\mu \mathrm{m}$ long; stalk cells cylindrical to cuneate, 5-10 $\mu \mathrm{m}$ long; head cells ovate to broadly ovate, attenuated and narrowed towards apex, entire, straight to curved, $15-17 \times 7-10 \mu \mathrm{m}$. Phialides mixed with appressoria, alternate, born on separate mycelial branch, ampulliform, 15-20 x 5-6 $\mu \mathrm{m}$. Perithecial setae 4-5 in numbers, straight, simple, acute to obtuse at the tip, often curved at the tip, up to $320 \mu \mathrm{m}$ long. Perithecia scattered, up to $90 \mu \mathrm{m}$ in diameter; ascospores oblong, 4-septate, slightly constricted at the septa, 40-45 x 12$20 \mu \mathrm{m}$.

This is the first species of the genus Irenopsis known on the members of the family Apocynaceae (Hansford 1961; Hosagoudar 1996, 2008; Hosagoudar et al. 1997; Hosagoudar \& Agarwal 2008; Hu et al. 1996, 1999).

\section{Irenopsis leeae Hansf. var. indica Hosag.}

Materials examined: TBGT 6435, PBL 028,TBGT 6438, PBL 31, 15.xii.2012, on leaves of Leea sp. (Leeaceae), Seeta nagar, Diglipur, North Andaman, coll. V.B. Hosagoudar et al.; TBGT 6443, PBL 036, 15.xii.2012, Kallara junction, Diglipur, North Andaman, coll. V.B. Hosagoudar et al.

\section{Irenopsis leeae Hansf. var. javensis Hansf.}

Material examined: TBGT 6509, PBL 102, 16.xii.2012, on leaves of Leea sp. (Leeaceae), Bakulthala, Middle Andaman, coll. V.B. Hosagoudar et al.; TBGT 6511, PBL 104, 16.xii.2012, Kallara junction, Diglipur, North Andaman, coll. V.B. Hosagoudar et al.
Irenopsis molleriana (Wint.) Stev.

Materials examined: TBGT 6484, PBL 077, 20.xii.2012, on leaves of Urena lobata (Malvaceae), Farrar gunj, South Andaman, coll. V.B. Hosagoudar et al.; TBGT 6519, PBL 112, 14.xii.2012, Hibiscus sp. ('Kattabhaji'), Lakshmipur nursery, Diglipur, North Andaman, coll. V.B. Hosagoudar et al.

The latter host (Kattabhaji) was growing profusely in most of the forest nurseries and according to locals, it is sour in taste and is being extensively used as vegetable after cooking with pulses. The infection was severe and probably this is the first report on this host plant.

\section{Irenopsis sidae (Rehm) Hughes var. indica Hosag. \& Manoj.}

Materials examined: TBGT 6457, PBL 050, TBGT 6483, 076, 20.xii.2012, on leaves of Sida sp. (Malvaceae), Farrar gunj, South Andaman, coll. V.B. Hosagoudar et al.

\section{Irenopsis triumfettae Stev. var. indica Hosag. \& Abraham}

Materials examined: TBGT 6420, PBL 013, 17.xii.2012, on leaves of Triumfetta sp. (Tiliaceae), Krishna Nagar, Baratang, Middle Andaman, coll. V.B. Hosagoudar et al.; TBGT 6485, PBL 078, 12.xii.2012, Dhanikari, South Andaman, coll. V.B. Hosagoudar et al.

\section{Meliola abri Hosag.}

Materials examined: TBGT 6454, PBL 47, 22.xii.2012, on leaves of Abrus precatorius (Fabaceae), BSI garden, Dhanikari, South Andaman, coll. V.B. Hosagoudar et al.; TBGT 6461, PBL 54, 20.xii.2012, Farrar gunj check post, South Andaman, coll. V.B. Hosagoudar et al.

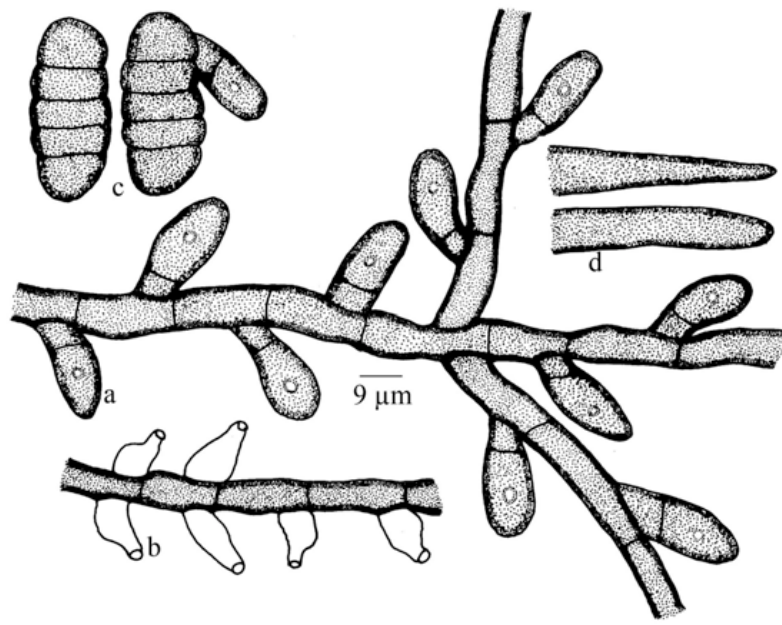

Figure 7. Irenopsis andamanica sp. nov.

a - Appressorium; b - Phialide; c - Ascospores; d - Perithecial setae 


\section{Meliola andamanica sp. nov.}

(Fig. 8) urn:Isid:indexfungorum.org:names: 807691

Materials examined: TBGT 6678 (holotype), PBL 272 (isotype), 17.xii.2012, on leaves of Strychnos andamanensis (Strychnaceae), Krishnanagar, Baratang, Middle Andaman, coll. V.B. Hosagoudar et al.

Colonies epiphyllous, subdense, scattered, up to $4 \mathrm{~mm}$ in diameter. Hyphae straight to substraight, branching mostly opposite at acute to wide angles, loosely to closely reticulate, cells $27-37 \times 5-7 \mu \mathrm{m}$. Appressoria opposite, $1 \%$ solitary, straight to curved, antrorse to subantrorse, 15-17 $\mu \mathrm{m}$ long; head cells ovate to oblong, entire, 10$12 \times 5-7 \mu \mathrm{m}$. Phialides mixed with appressoria, alternate to opposite, ampulliform, 20-25x5-7 $\mu \mathrm{m}$. Mycelial setae scattered, simple, straight, acute to obtuse at the tip, up to $750 \mu \mathrm{m}$ long. Perithecia scattered, globose, up to $180 \mu \mathrm{m}$ in diameter; ascospores obovoidal, 4-septate, deeply constricted at the septa, 37-40x12-15 $\mu \mathrm{m}$.

This is the only species of the genus Meliola known in the member of the family Strychnaceae in having only opposite appressoria (some are solitary) (Hansford 1961; Hosagoudar 1996, 2008, 2013; Hue et al. 1997).

Etymology: Named after Andaman Islands.

\section{Meliola anceps Sydow \& Sydow}

Materials examined: TBGT 6412, PBL 005, 18.xii.2012, on leaves of Mussaenda philippica (Rubiaceae), Lorogic forest camp, Baratang, Middle Andaman, coll. V.B. Hosagoudar et al.

\section{Meliola butleri Sydow \& Sydow}

Materials examined: 14.xii.2012, TBGT 6533, PBL 126, on leaves of Citrus sp. (Rutaceae), Lakshmipur Nursery, North Andaman, coll. V.B. Hosagoudar et al.

The infection was very severe on this cultivated plant.

\section{Meliola canarifolia sp. nov.}

(Fig. 9) urn:Isid:indexfungorum.org:names: 807692

Materials examined: TBGT 6684 (holotype), PBL 278 (isotype), 21.xii.2012, on leaves of Canarium sp. (Burseraceae), Tusnabad, South Andaman, coll. V.B. Hosagoudar et al.

Colonies amphigenous, scattered, dense, up to $4 \mathrm{~mm}$ in diameter. Hyphae substraight to flexuous, branching opposite at acute to wide angles, loosely to closely reticulate, cells $35-42 \times 6-10 \mu \mathrm{m}$. Appressoria alternate to unilateral, straight to curved, closely antrorse to subantrorse, 22-32 $\mu \mathrm{m}$ long; stalk cells cylindrical to cuneate, 5-10 $\mu \mathrm{m}$ long; head cells, ovate, oblong,

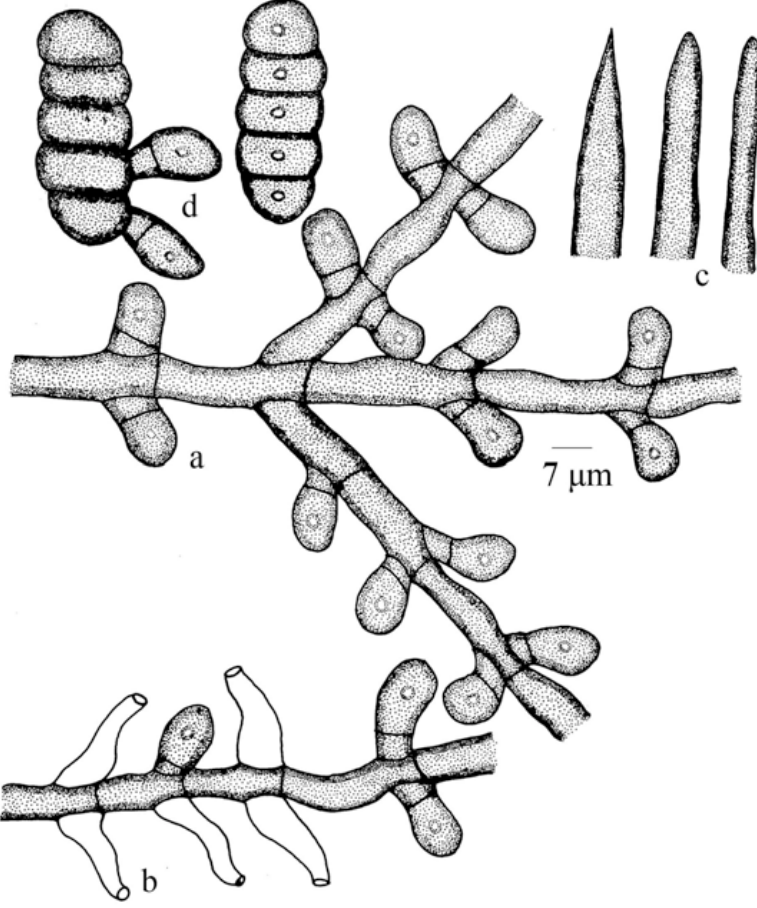

Figure 8. Meliola andamanica sp. nov.

a - Appressorium; b - Phialide; c - Apical portion of the mycelial setae; d - Ascospores

clavate, often cylindrical, entire to rarely sublobate, truncate, straight to curved, $12-25 \times 10-15 \mu \mathrm{m}$. Phialides mixed with appressoria, alternate, ampulliform, 2230x6-7 $\mu \mathrm{m}$ long. Mycelial setae scattered, simple, straight, acute to obtuse at the tip, up to $400 \mu \mathrm{m}$ long; mycelial setae around perithecia were simple, straight, curved, uncinate at the tip, up to $300 \mu \mathrm{m}$ long. Perithecia scattered, up to $210 \mu \mathrm{m}$ in diameter; ascospores oblong to cylindrical, 4-septate, mostly constricted at the septa, 47-55x15-20 $\mu \mathrm{m}$.

Meliola canarii is known on this host genus from the tropical countries and also from the Western Ghats of Karnataka and Tamil Nadu (Hansford 1961; Hosagoudar 1996). However, Meliola canarifolia differs from Meliola canarii in having frequently truncate and rarely sublobate head cells of the appressoria and distinctly larger ascospores.

Etymology: Named after its host plant.

Meliola careyae (Stev.) Hosag. var. indica Hosag.

Materials examined: TBGT 6453, PBL 46, 22.xii.2012, on leaves of Careya arborea (Lecythidaceae), BSI garden, Dhanikari, South Andaman, coll. V.B. Hosagoudar et al. 


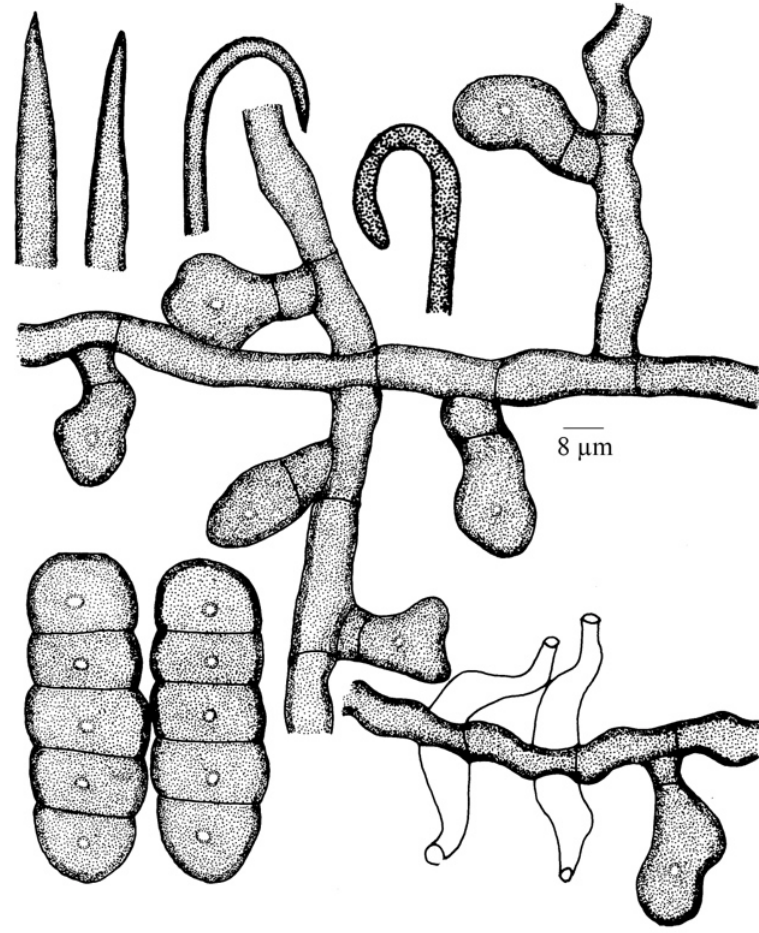

Figure 9. Meliola canarifolia sp. nov.

a - Appressorium, b - Phialide, c - Apical portion of the mycelial setae; d - Ascospores

\section{Meliola cadambae Hosag. \& C.K. Biju}

Materials examined: TBGT 6411, PBL 004, 17.xii.2012, on leaves of Neolamarkia cadamba (Rubiaceae), Krishna nagar, Baratang, Middle Andaman, coll. V.B. Hosagoudar et al.; TBGT 6429, PBL 022, 17.xii.2012, South creek, Baratang, Middle Andaman, coll. V.B. Hosagoudar et al.; TBGT 6439, PBL 032, 15.xii.2012, Seeta nagar, Diglipur, North Andaman, coll. V.B. Hosagoudar et al.

This is very common plant along with the edges of the evergreen forests with this fungus.

\section{Meliola citricola Sydow \& Sydow}

Materials examined: TBGT 6424, PBL 17, 18.xii.2012, on leaves of Citrus sp. (Rutaceae), Gandhi Ghat, Baratang, Middle Andaman, coll. V.B. Hosagoudar et al.; TBGT 6427, PBL 20, 18.xii.2012, Gandhi Ghat, Baratang, Middle Andaman, coll. V.B. Hosagoudar et al.; TBGT 6433, PBL 26, 15.xii.2012, Kalpong hydroelectric project area, Nabagram, Diglipur, North Andaman, coll. V.B. Hosagoudar et al.; TBGT 6475, PBL 68, 14.xii.2012, Radhanagar, Diglipur, North Andaman, coll. V.B. Hosagoudar et al.
Meliola chukrasiicola sp. nov.

(Fig. 10) urn:Isid:indexfungorum.org:names: 807693

Materials examined: TBGT 6681 (holotype), PBL 275 (isotype), 8.xii.2012, on leaves of Chukrasia sp. (Meliaceae), Central nursery, Lorogic Forest Camp, Middle Andaman, coll. V.B Hosagoudar et al.

Colonies epiphyllous, thin, up to $6 \mathrm{~mm}$ in diameter, confluent. Hyphae straight to substraight, branching opposite to irregular at acute to wide angles, loosely to closely reticulate, cells $22-32 \times 6-7 \mu \mathrm{m}$. Appressoria alternate, rarely unilateral, antrorse, sub antrorse to retrorse, straight to curved, 15-22 $\mu \mathrm{m}$ long; stalk cells cylindrical to cuneate, 5-7 $\mu \mathrm{m}$ long; head cells ovate, globose, clavate, entire, straight to curved, 10-16x7$10 \mu \mathrm{m}$. Phialides mixed with appressoria, alternate to opposite, ampulliform, 17-22x5-6 $\mu \mathrm{m}$. Mycelial setae loosely scattered, straight, simple, acute to 2-3-dentate at the tip, up to $650 \mu \mathrm{m}$ long. Perithecia scattered, up to $170 \mu \mathrm{m}$ in diameter; ascospores cylindrical, 4- septate, constricted at the septa, 40-47x15-17 $\mu \mathrm{m}$.

Meliola chukrasiae known on Chukrasia sp. from Western Ghats (Hosagoudar 1996). However, Meliola chukrasiicola differs from it in having straight, acute to 2-3-dentate mycelial setae.

Etymology: Named after its host plant.

\section{Meliola clerodendricola Henn.}

Materials examined: TBGT 6444, PBL 037, 20.xii.2012, on leaves of Clerodendrum sp. (Verbenaceae), Farrar gunj, South Andaman, coll. V.B. Hosagoudar et al.; TBGT 6512, PBL 105, 16.xii.2012, Betapur, Middle Andaman, coll. V.B. Hosagoudar et al.; TBGT 6501, PBL 094, 7.xii.2012, Dhanikari, South Andaman, coll. V.B. Hosagoudar et al.; TBGT 6494, PBL 087, 8.xii.2012, Sholbay 14, South Andaman, coll. V.B. Hosagoudar et al.; TBGT 6481, PBL 074, 12.xii.2012, Dhanikari, South Andaman, coll. V.B. Hosagoudar et al.; TBGT 6446, PBL 039, 20.xii.2012, Farrar gunj, South Andaman, coll. V.B. Hosagoudar et al.; TBGT 6468, PBL 061, 8.xii.2012, C. viscosum, Sholbay 14, South Andaman, coll. V.B. Hosagoudar et al.

\section{Meliola entadicola Deight.}

Materials examined: TBGT 6455, PBL 48, 22.xii.2012, on the pods of Entada sp. (Mimosaceae), BSI garden, Dhanikari, South Andaman, coll. V.B. Hosagoudar et al.; TBGT 6465, PBL 58, 15.xii.2012, Kallara junction, Diglipur, North Andaman, coll. V.B. Hosagoudar et al. 


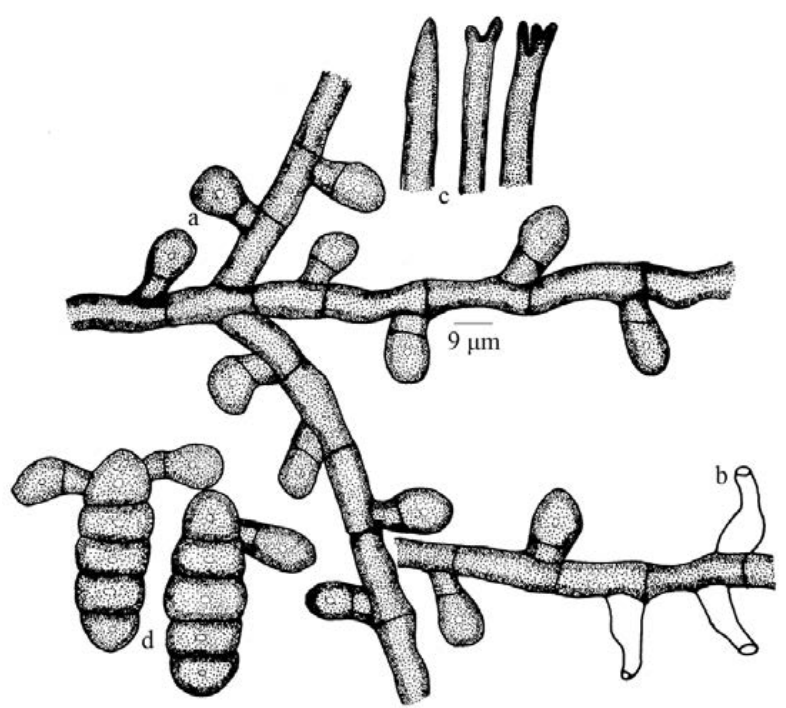

Figure 10. Meliola chukrasiicola sp. nov.

a - Appressorium, b - Phialide, c - Apical portion of the mycelial setae; d - Ascospores

Meliola flemingiicola Hosag. et al.

Materials examined: TGBT 6423, PBL 16, 18.xii.2012, on leaves of Flemingia sp. (Fabaceae), Gandhi Nagar, Middle Andaman, coll. V.B. Hosagoudar et al.; TBGT 6456, PBL 49, 21.xii.2012, Tushnabad, South Andaman, coll. V.B. Hosagoudar et al.

Meliola gliricidiicola Hosag. \& Agarwal

Materials examined: TBGT 6469, PBL 62, 8.xii.2012, on leaves of Gliricidia sepium (Fabaceae), riverside, Wrighte Myo, South Andaman, coll. V.B. Hosagoudar et al.; TBGT 6534, PBL 127, 16.xii.2012, Bettapur, Middle Andaman, coll. V.B. Hosagoudar et al.; TBGT 6527, PBL 120, 13.xii.2012, Tugapur rest camp, Diglipur, North Andaman, V.B. Hosagoudar et al.; TBGT 6487, PBL 80, 20.xii.2012, Farrar gunj, South Andaman, coll. V.B. Hosagoudar et al.

Meliola harrietensis sp. nov.

(Fig. 11) urn:Isid:indexfungorum.org:names: 807694

Materials examined: TBGT 6672 (holotype), PBL 265 (isotype), PBL 266, 11.xii.2012, on leaves of Diospyros ridli (Ebenaceae), Mount Harriet National Park, South Andaman, coll. V.B. Hosagoudar et al.

Colonies mostly hypophyllous, scattered, dense, up to $4 \mathrm{~mm}$ in diameter. Hyphae straight to substraight, branching opposite to irregular at acute to wide angles, loosely to closely reticulate, cells $20-25 \times 7-10 \mu \mathrm{m}$. Appressoria opposite, 2-3\% alternate to rarely solitary, straight to curved, closely antrorse, subantrorse to retrorse, 12-22 $\mu \mathrm{m}$ long; stalk cells cylindrical to cuneate, 5-7 $\mathrm{m}$ long; head cells ovate to oblong, cylindrical, entire, straight to curved, $7-17 \times 7-10 \mu \mathrm{m}$. Phialides mixed with appressoria, alternate, ampulliform, 2032x5-7 $\mu \mathrm{m}$. Mycelial setae densely scattered, simple, acute to obtuse, uncinate to coiled at the tip, up to $250 \mu \mathrm{m}$ long. Perithecia scattered, up to $170 \mu \mathrm{m}$ in diameter; ascospores oblong to cylindrical, 4-septate, mostly constricted at the septa, $37-45 \times 15-17 \mu \mathrm{m}$.

This species is distinct from all other Meliola species known on the members of family Ebenaceae in having 2-5\% alternate appressoria and uncinate to coiled mycelial setae (Hansford 1961; Hosagoudar 1996, 2008, 2013).

Etymology: Named after collection locality.

\section{Meliola hemidesmicola Hosag.}

Materials examined: TBGT 6515, PBL 108, 11.xii.2012, on leaves of Hemidesmus sp. (Periplocaceae), Mount Harriet National Park, South Andaman, coll. V.B. Hosagoudar et al.

\section{Meliola hyptidis Sydow}

Materials examined: TBGT 6417, PBL 10, 18.xii.2012, on leaves of Hyptis sp. (Lamiaceae), Lorogic forest camp, Baratang, Middle Andaman, coll. V.B. Hosagoudar et al.; TBGT 6426, PBL 19, 18.xii.2012, Gandhi nagar, Middle Andaman, coll. V.B. Hosagoudar et al.; TBGT 6440, PBL 33, 15.xii.2012, Seeta nagar, Diglipur, North Andaman, coll. V.B. Hosagoudar et al.; TBGT 6514, PBL 107, 16.xii.2012, Bakulthala, Middle Andaman, coll. V.B. Hosagoudar et al.; TBGT 6506, PBL 99, 16.xii.2012, Panighat, Diglipur, North Andaman, V.B. Hosagoudar et al.; TBGT 6495, PBL 88, 8.xii.2012, Sholbay 14, South Andaman, V.B. Hosagoudar et al.; TBGT 6478, PBL 71, 14.xii.2012, Radhanagar, Diglipur, North Andaman, coll. V.B. Hosagoudar et al.; TBGT 6464, PBL 57, 8.xii.2012, Sholbay 14, South Andaman, coll. V.B. Hosagoudar et al.; TBGT 6462, PBL 55, 8.xii.2012, Sholbay 14, South Andaman, coll. V.B. Hosagoudar et al.; TBGT 6449, PBL 42, 21.xii.2012, Tushnabad, South Andaman, coll. V.B. Hosagoudar et al.; TBGT 6442, PBL 35, 15.xii.2012, Kallara junction, Diglipur, North Andaman, V.B. Hosagoudar et al.

\section{Meliola jasmini Hansf. \& Stev.}

Materials examined: TBGT 6474 PBL 67, 14.xii.2012, on leaves of Jasminum sambac (Oleaceae), Shibpur, Diglipur, North Andaman, coll. V.B. Hosagoudar et al.; TBGT 6526 PBL 119, 14.xii.2012, Shamnagar, Diglipur, North Andaman, coll. V.B. Hosagoudar et al. 


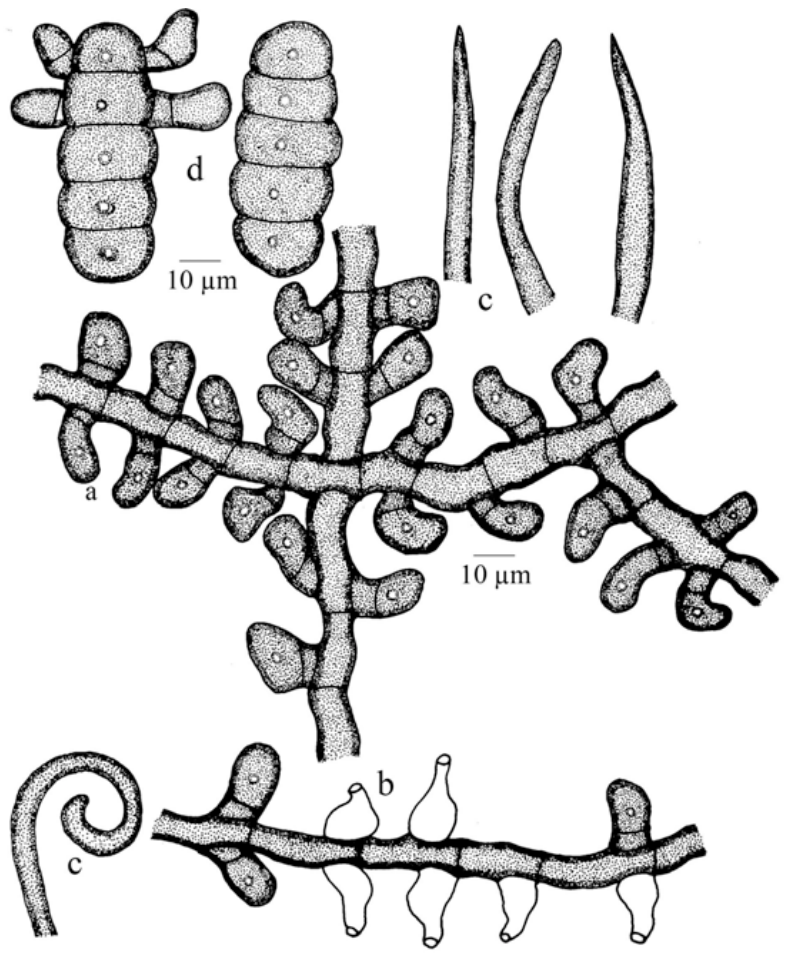

Figure 11. Meliola harrietensis sp. nov.

a - Appressorium, b - Phialide, c - Apical portion of the mycelial setae; d - Ascospores

Meliola palmicola Wint. (Fig. 12 \& Image 2)

Materials examined: TBGT 6683, PBL 277; TBGT 6685, PBL 279; TBGT 6687, PBL 281; TBGT 6689, PBL 283, 14.xii.2012, on leaves of Licuala peltata (Arecaceae), Krishnanagar, Baratang, Middle Andaman, coll. V.B. Hosagoudar et al.

Colonies epiphyllous, subdense to dense, up to $3 \mathrm{~mm}$ in diameter, confluent. Hyphae straight to substraight, branching opposite to irregular, at acute to wide angles, loosely to closely reticulate, cells $37-50 \times 7-10$ $\mu \mathrm{m}$. Appressoria alternate to unilateral, antrorse to sub antrorse, straight to curved, 30-40 $\mu \mathrm{m}$ long; stalk cells cylindrical to cuneate, 7-17 $\mu \mathrm{m}$ long; head cells ovate, oblong, cylindrical, often hammer shaped, entire. angular, to sublobate, straight to curved, 17-22×12-20 $\mu \mathrm{m}$. Phialides apparently born on a separate mycelial branch but are often mixed with appressoria, alternate, ampulliform, 17-25x6-8 $\mu \mathrm{m}$. Mycelial setae scattered, simple, straight, flexuous, geniculate, acute, obtuse, variously dentate, furcate to slightly branched at the tip, up to $1300 \mu \mathrm{m}$ long. Perithecia scattered, globose, up to $240 \mu \mathrm{m}$ in diameter; ascospores cylindrical, 4-septate, slightly constricted at the septa, $80-85 \times 20-25 \mu \mathrm{m}$.
Meliola malacotricha Speg.

Materials examined: TBGT 6414, PBL 7, 18.xii.2012, on leaves of Argyreia sp. (Convolvulaceae), Gandhi nagar, Middle Andaman, coll. V.B. Hosagoudar et al.; TBGT 6416 PBL 9, 17.xii.2012, South Creek, Baratang, Middle Andaman, coll. V.B. Hosagoudar et al.; TBGT 6421 PBL 14, 18.xii.2012, Near Central nursery, Lorogic forest camp, Baratang, Middle Andaman, coll. V.B. Hosagoudar et al.; TBGT 6431 PBL 24, 11.xii.2012, Mount Harriet, South Andaman, coll. V.B. Hosagoudar et al.; TBGT 6436 PBL 29, 20.xii.2012, Farrar gunj, South Andaman, coll. V.B. Hosagoudar et al.; TBGT 6500 PBL 93, 8.xii.2012, Sholbay 14, South Andaman, coll. V.B. Hosagoudar et al.

\section{Meliola malacotricha Speg. var. major Beeli}

Materials examined: TBGT 6517 PBL 110, 14.xii.2012, on leaves of Argyreia sp. (Convolvulaceae), Near Lakshmipur nursery, Diglipur, North Andaman, coll. V.B. Hosagoudar et al.; TBGT 6503 PBL 96, 8.xii.2012, Sholbay 14, South Andaman, coll. V.B. Hosagoudar et al.; TBGT 6441 PBL 34, 15.xii.2012, Merremia sp., Seeta

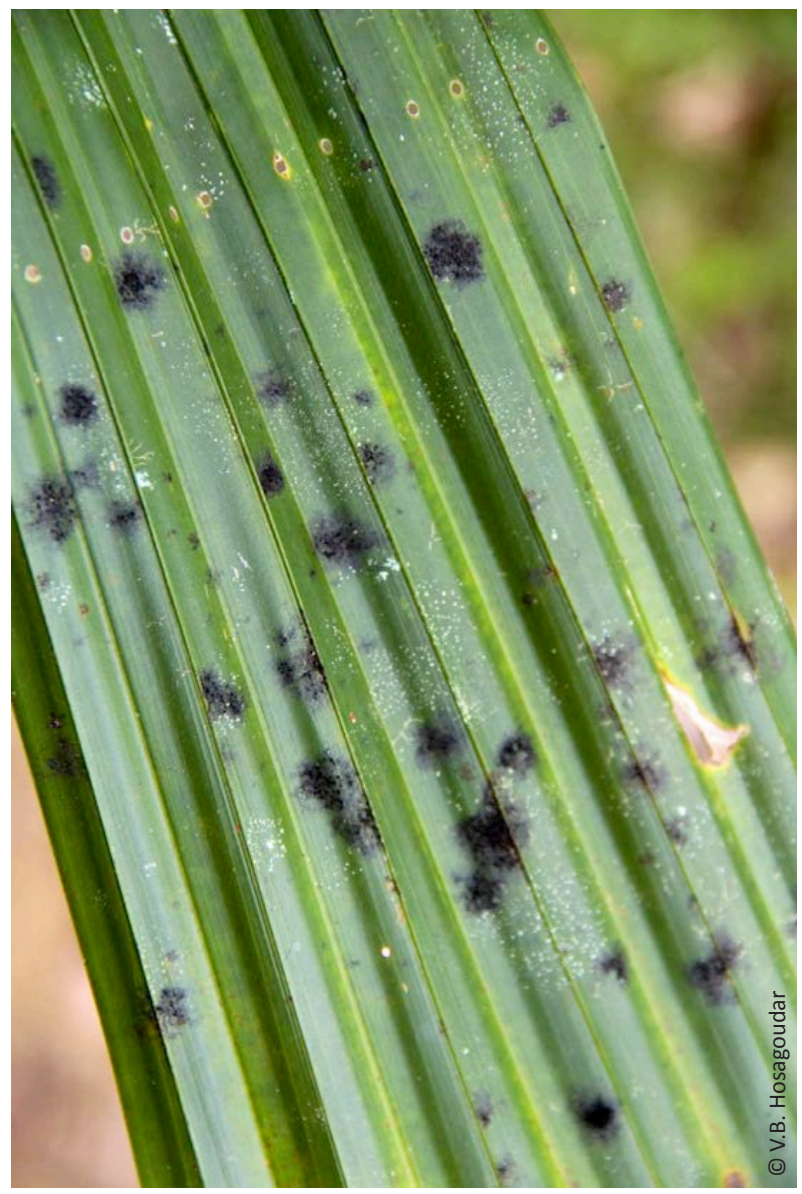

Image 2. Meliola palmicola Wint. - Infected leaf 


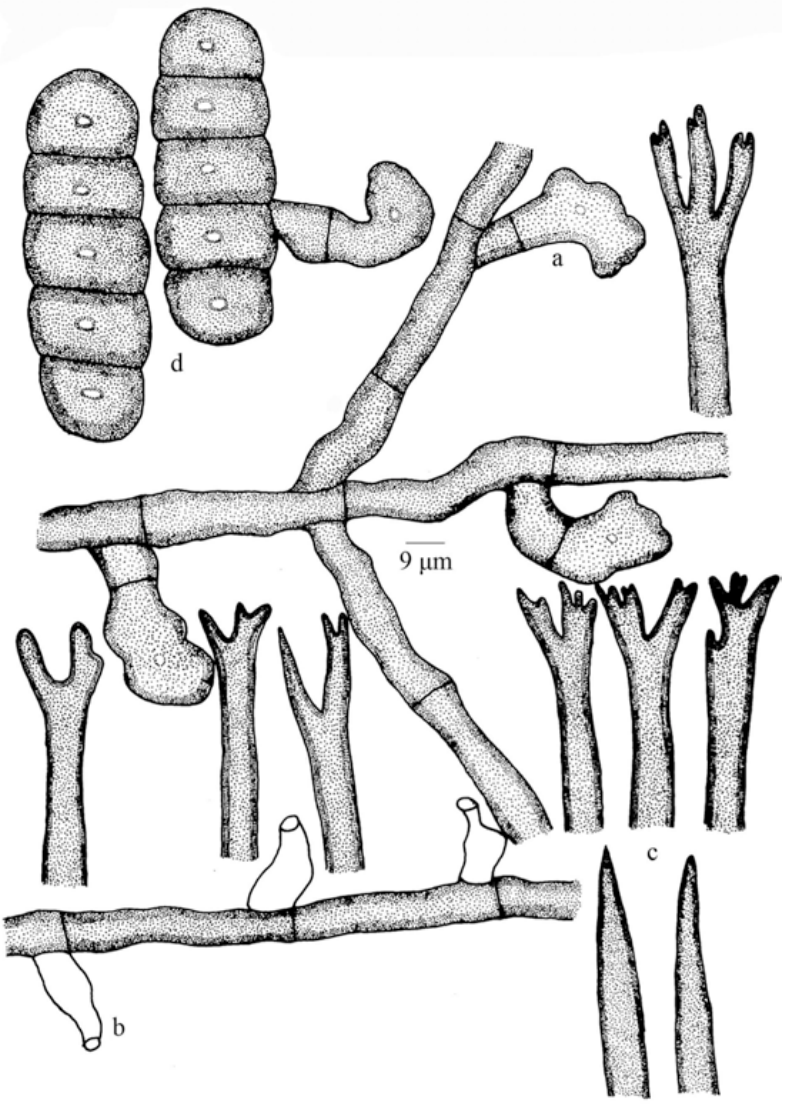

Figure 12. Meliola palmicola Wint.

a - Appressorium, b - Phialide, c - Apical portion of the mycelial setae; d - Ascospores

nagar, Bamboo tikiri, Diglipur, North Andaman, coll. V.B. Hosagoudar et al.; TBGT 6419 PBL 12, 17.xii.2012, Argyreia sp., Jarwa Creek, Baratang, Middle Andaman, coll. V.B. Hosagoudar et al.; TBGT 6529 PBL 122, 8.xii.2012, Wright Myo, riverside, South Andaman, coll. V.B. Hosagoudar et al.

\section{Meliola mangiferae Earle}

Materials examined: TBGT 6413 PBL 6, 18.xii.2012, on leaves of Mangifera indica (Anacardiaceae), Gandhi nagar, Middle Andaman, coll. V.B. Hosagoudar et al.; TBGT 6415 PBL 8, 18.xii.2012, Adagic, Baratang, Middle Andaman, coll. V.B. Hosagoudar et al.; TBGT 6422 PBL 15, 18.xii.2012, Gandhi ghat, Baratang, Middle Andaman, coll. V.B. Hosagoudar et al.; TBGT 6599 PBL 92, 20.xii.2012, Farrar gunj, South Andaman, coll. V.B. Hosagoudar et al.; TBGT 6492 PBL 85, 15.xii.2012, Central forest nursery, Nabagram, Diglipur, North Andaman, coll. V.B. Hosagoudar et al.; TBGT 6479 PBL 72, 14.xii.2012, Near Lakshmipur nursery, Diglipur, North Andaman, coll. V.B. Hosagoudar et al.; TBGT 6477 PBL 70, 14.xii.2012, Radhanagar, Diglipur, North Andaman, coll.
V.B. Hosagoudar et al.; TBGT 6471 PBL 64, 14.xii.2012, Diglipur, North Andaman, coll. V.B. Hosagoudar et al.; TBGT 6467 PBL 60, 14.xii.2012, Dhankari, South Andaman, coll. V.B. Hosagoudar et al.; TBGT 6450 PBL 43, 21.xii.2012, Tushnabad, South Andaman, coll. V.B. Hosagoudar et al.; TBGT 6507 PBL 97, 11.xii.2012, Mangifera camptosperma, Mount Harriet, South Andaman, coll. V.B. Hosagoudar et al.; TBGT 6498, PBL 91, 11.xii.2012, Mount Harriet, South Andaman, coll. V.B. Hosagoudar et al.; TBGT 6466, PBL 59, 11.xii.2012, Mount Harriet, South Andaman, coll. V.B. Hosagoudar et al.

\section{Meliola melanoxylonis Hosag. \& Pillai}

Materials examined: TBGT 6530, PBL 123, 8.xii.2012, on leaves of Acacia auriculiformis (Mimosaceae), Sholbay 14, South Andaman, coll. V.B. Hosagoudar et al.

\section{Meliola myristicacearum sp. nov.}

(Fig. 13) urn:Isid:indexfungorum.org:names: 807695

Materials examined: TBGT 6690 (holotype), PBL 284 (isotype), 11.xii.2012, on leaves of Myristicaceae member, Mount Harriet National Park, South Andaman, coll. V.B. Hosagoudar et al.

Colonies epiphyllous, scattered, dense, up to $5 \mathrm{~mm}$ in diameter, confluent. Hyphae straight, substraight to flexuous, branching opposite to irregular at acute to wide angles, closely reticulate, cells 25-35x7-8 $\mu \mathrm{m}$. Appressoria opposite to alternate, straight to curved, antrorse to subantrorse, 20-30 $\mu \mathrm{m}$ long; stalk cells cylindrical to cuneate, 7-12 $\mu \mathrm{m}$ long; head cells, ovate, oblong to globose, clavate, entire, broadly rounded to truncate at the apex, straight to curved, 12-17 x 10$12 \mu \mathrm{m}$. Phialides mixed with appressoria, opposite to alternate, ampulliform, 17-25x6-10 $\mu \mathrm{m}$ long. Mycelial setae scattered, simple, straight to uncinate, acute to obtuse at the tip, up to $460 \mu \mathrm{m}$ long. Perithecia scattered, up to $200 \mu \mathrm{m}$ in diameter; ascospores oblong to cylindrical, 4-septate, mostly constricted at the septa, 45-50x17-20 $\mu \mathrm{m}$.

Meliola knemae Hosag. \& Robin is known on Knema attenuata from the Western Ghats of Kerala. However, the present species difers from it in having opposite appressoria and uncinate mycelial setae (Hosagoudar \& Robin 2011).

Etymology: Named after the family of host plant.

\section{Meliola panici Earle}

Materials examined: TBGT 6430, PBL 23, 21.xii.2012, on leaves of Poaceae (Grass) member, Tushnabad, South 
Andaman, coll. V.B. Hosagoudar et al.; TBGT 6505, PBL 98, 11.xii.2012, Oplismenus sp., Mount Harriet, South Andaman, coll. V.B. Hosagoudar et al.

\section{Meliola parishiae sp. nov.}

(Fig. 14) urn:Isid:indexfungorum.org:names: 807703

Materials examined: TBGT 6674 (holotype), PBL 268 (isotype), 8.xii.2012, on leaves of Parishia insignis (Anacardiaceae), Wright Myo, South Andaman, coll. V.B. Hosagoudar et al.

Colonies amphigenous, scattered, thin, up to $3 \mathrm{~mm}$ in diameter. Hyphae straight to substraight, branching opposite at acute to wide angles, loosely to closely reticulate, cells 27-35x5-7 $\mu \mathrm{m}$. Appressoria opposite to unilateral, straight to curved, antrorse to subantrorse, 25-30 $\mu \mathrm{m}$ long; stalk cells cylindrical to cuneate, 5-10 $\mu \mathrm{m}$ long; head cells ovate, obovate, entire, straight to curved, 17-22x6-7 $\mu \mathrm{m}$. Phialides mixed with appressoria, opposite to alternate, ampulliform, 17-25x6-7 $\mu \mathrm{m}$. Mycelial setae scattered, simple, straight, acute to 2-3 dentate at the tip, up to $300 \mu \mathrm{m}$ long. Perithecia scattered, up to $180 \mu \mathrm{m}$ in diameter; ascospores oblong

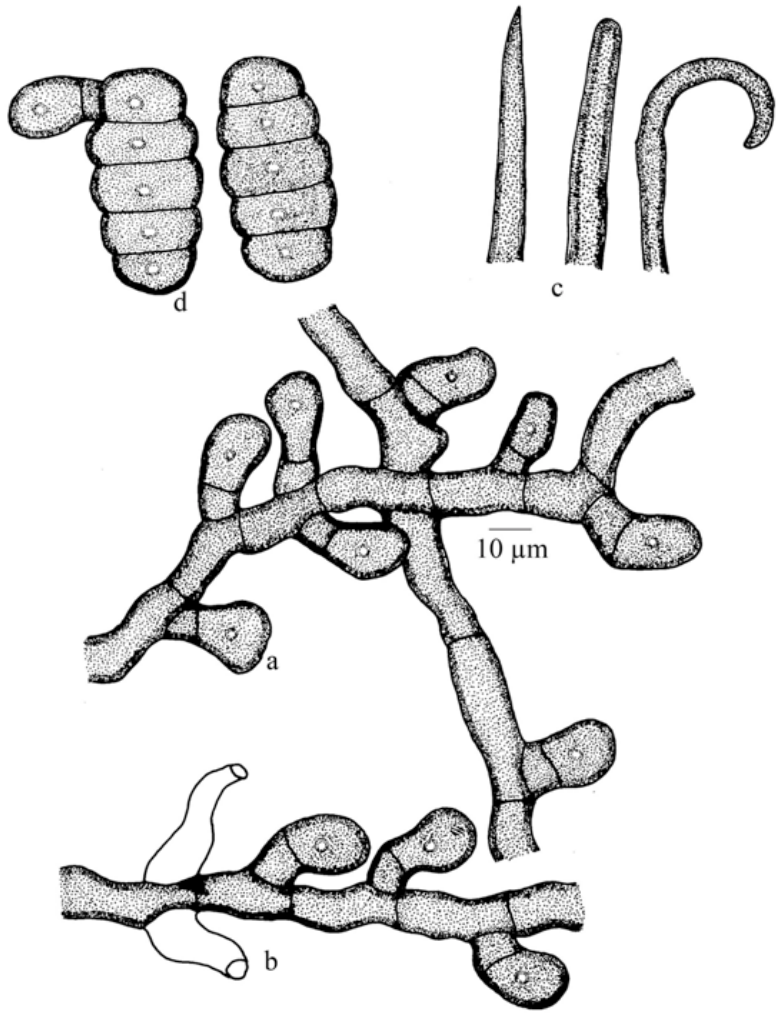

Figure 13. Meliola myristicacearum sp. nov.

a - Appressorium, b - Phialide, c - Apical portion of the mycelial setae; d-Ascospores to cylindrical, 4-septate, mostly constricted at the septa, 40-45x15-17 $\mu \mathrm{m}$.

Meliola parishiae differs from all the known Meliola member of the family Ancardiaceae in having entire head cells of the appressoria, acute, obtuse to 2-3 dentate and straight to uncinate mycelia setae (Hansford 1961; Hosagoudar 1996, 2008, 2013; Hue et al. 1996, 1999).

\section{Meliola pithecellobii var.indica var. nov.}

(Fig. 15) urn:Isid:indexfungorum.org:names: 807707

Materials examined: TBGT 6665 (holotype), PBL 258 (isotype), 8.xii.2012, on leaves of Pithecellobium sp. (Mimosaceae), Wright Myo, South Andaman, coll. V.B. Hosagoudar et al.

Colonies epiphyllous, thin, up to $3 \mathrm{~mm}$ in diameter. Hyphae straight to substraight, branching alternate to opposite at acute to wide angles, loosely reticulate, cells 23-30x5-7 $\mu \mathrm{m}$. Appressoria mostly unilateral, rarely opposite (2\%), straight to curved, mostly perpendicular to the hyphae, antrorse, subantrorse to retrorse, 10-17 $\mu \mathrm{m}$ long; stalk cells cylindrical to cuneate, $2-7 \mu \mathrm{m}$ long; head cells globose, ovate, straight to curved, broadly rounded to truncate, often pointed to the side, entire, 7-11x7-10 $\mu \mathrm{m}$. Phialides mixed with appressoria, alternate to opposite, ampulliform, 20-25x7-10 $\mu \mathrm{m}$ long. Mycelial setae scattered, straight, simple, acute

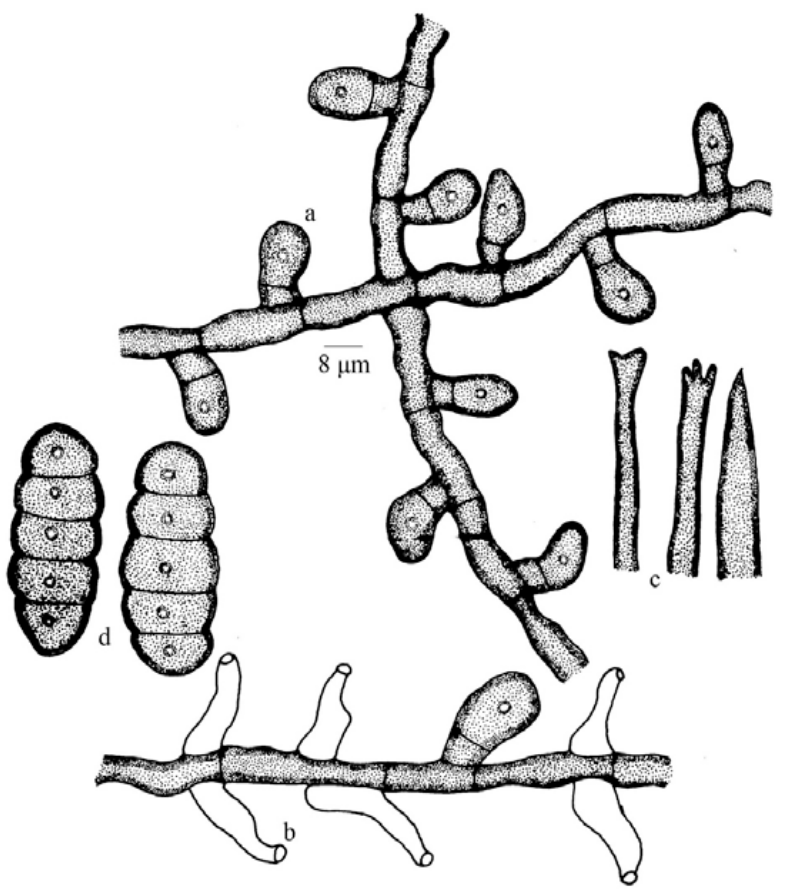

Figure 14. Meliola parishiae sp. nov.

a - Appressorium, b - Phialide, c - Apical portion of the mycelial setae; d - Ascospores 
to dentate at the tip, up to $450 \mu \mathrm{m}$ long. Perithecia scattered, globose, up to $190 \mu \mathrm{m}$ in diameter; ascospores obovoidal, 4-septate, mostly constricted at the septa, 35-40x10-15 $\mu \mathrm{m}$.

Meliola pithecellobii is known on pithecellobium species from Jawa and Trinidad (Hansford 1961). However, differs from the var. pithecellobii in having simple to dentate mycelia setae. This taxon also differs from another variety of this species uncinata Bezerra Gadelha (Hosagoudar et al. 1997) in having only straight mycelial setae in contrast to uncinate setae.

\section{Meliola pterocarpi Yates}

Materials examined: TBGT 6425 PBL 18, 20.xii.2012, on leaves of Pterocarpus dalbergiodes (Fabaceae), Farrar gunj, South Andaman, coll. V.B. Hosagoudar et al.; TBGT 6532 PBL 125, 14.xii.2012, Lakshmipur Nursery, North Andaman, coll. V.B. Hosagoudar et al.; TBGT 6507 PBL 100, 14.xii.2012, Millengram, Diglipur, North Andaman, coll. V.B. Hosagoudar et al.; TBGT 6491 PBL 84, 15.xii.2012, Bamboo tikiri, Diglipur, North Andaman, coll. V.B. Hosagoudar et al.

\section{Meliola raulfiae Mibey \& Hawksworth}

Materials examined: TBGT 6463, PBL 56, 8.xii.2012, on leaves of Rauvolfia tetraphylla (Apocynaceae), Mannarcaud, South Andaman, coll. V.B. Hosagoudar et al.

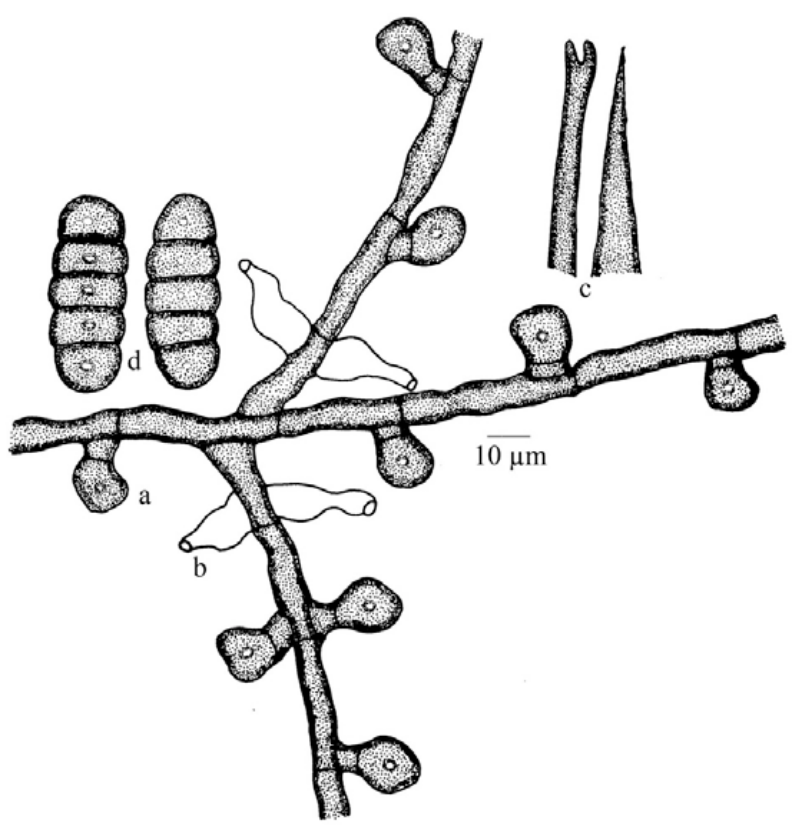

Figure 15. Meliola pithecellobii var. indica var. nov.

a - Appressorium, b - Phialide, c - Apical portion of the mycelial setae; d - Ascospores
Meliola savarkarii sp. nov.

(Fig. 16) urn:Isid:indexfungorum.org:names: 807704

Materials examined: TBGT 6664 (holotype), PBL 257 (isotype), 8.xii.2012, on leaves of Aglaia sp. (Meliaceae), Sholbay, South Andaman, coll. V.B. Hosagoudar et al.

Colonies epiphyllous, scattered, thin, up to $2 \mathrm{~mm}$ in diameter, confluent. Hyphae substraight, flexuous to slightly crooked, branching mostly opposite at wide angles, loosely to closely reticulate, cells 20-30x5-7 $\mu \mathrm{m}$. Appressoria alternate, less than $1 \%$ opposite to unilateral, straight to slightly curved, subantrorse to retrorse, often spreading, 15-22 $\mu \mathrm{m}$ long; stalk cells cylindrical to cuneate, 5-7 $\mu \mathrm{m}$ long; head cells ovate, clavate to oblong, entire, rarely truncate, straight to curved, 10$15 \times 6-8 \mu \mathrm{m}$. Phialides mixed with appressoria, alternate to opposite, ampulliform, 12-17x5-8 $\mu \mathrm{m}$ long. Mycelial setae few, simple, straight, acute, obtuse to $2-5$ cristate at the tip, up to $260 \mu \mathrm{m}$ long. Perithecia scattered, up to $190 \mu \mathrm{m}$ in diameter; ascospores ellipsoidal to cylindrical, 4-septate, constricted at the septa, 35-42×12-17 $\mu \mathrm{m}$.

This species is similar to Meliola silentvalleyensis Hosag. but differs from it in having phialides mixed with appressoria and head cells of the appressoria are entire (Hosagoudar 2008).

Etymology: Named after Veer Savarkar, a freedom fighter

\section{Meliola tamarindi Sydow \& Sydow}

Materials examined: TBGT 6409 PBL 2, 18.xii.2012, on leaves of Tamarindus indica (Caesalpiniaceae), Central nursery, Baratang, Middle Andaman, coll. V.B. Hosagoudar et al.

\section{Meliola ternstroemiicola sp. nov.}

(Fig. 17) urn:Isid:indexfungorum.org:names: 807705

Materials examined: TBGT 6668 (holotype), PBL 261 (isotype), 11.xii.2012, on leaves of Ternstroemia vahliana (Theaceae), Mount Harriet National Park, South Andaman, coll. V.B. Hosagoudar et al.

Colonies hypophyllous, subdense, spreading, up to $5 \mathrm{~mm}$ in diameter, confluent. Hyphae straight to substraight, branching opposite to alternate at acute angles, loosely reticulate, cells $22-27 \times 6-7 \mu \mathrm{m}$. Appressoria alternate to unilateral, straight to curved, antrorse, subantrorse to retrorse, 17-25 $\mu \mathrm{m}$ long; stalk cells cylindrical to cuneate, 5-7 $\mu \mathrm{m}$ long; head cells ovate to oblong, globose, entire, straight to curved, 10-17x6$8 \mu \mathrm{m}$. Phialides mixed with appressoria, opposite to alternate, ampulliform, variously curved, 20-25x5-7 $\mu \mathrm{m}$ 


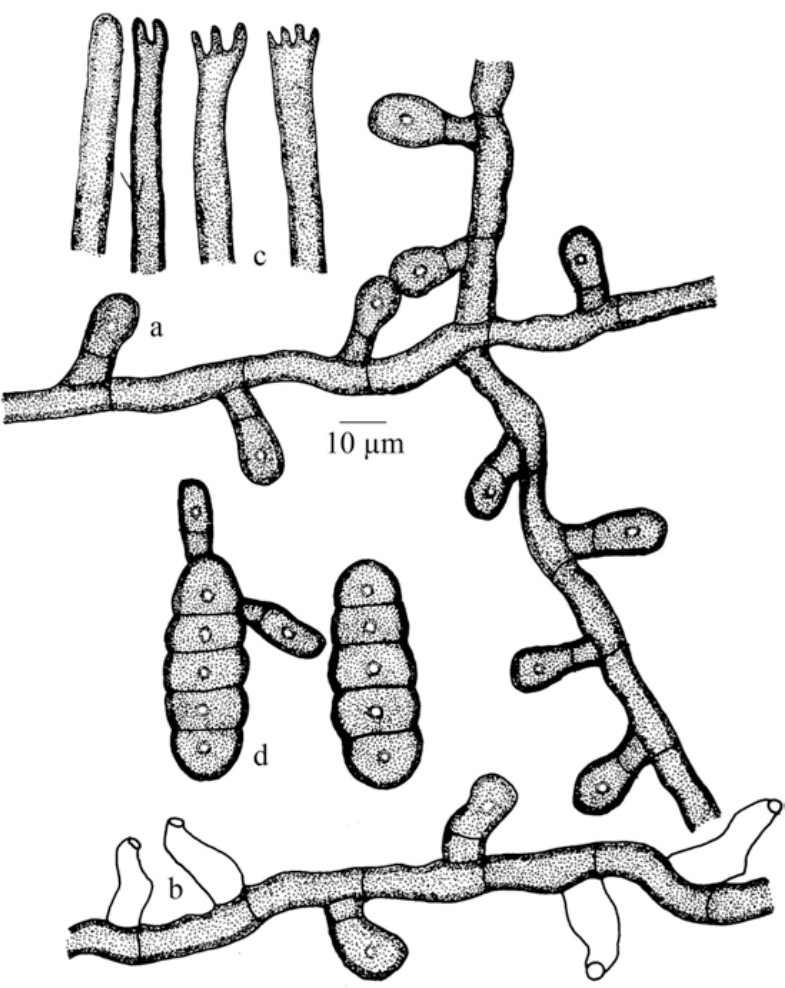

Figure 16. Meliola savarkarii sp. nov.

a - Appressorium, b - Phialide, c - Apical portion of the mycelial setae; d - Ascospores

long. Mycelial setae scattered, simple, straight, acute to obtuse, variously dentate to cristate at the tip, up to $1450 \mu \mathrm{m}$. Perithecia scattered, up to $120 \mu \mathrm{m}$ in diameter; ascospores oblong to cylindrical, 4-septate, constricted at the septa, $42-47 \times 15-20 \mu \mathrm{m}$.

Amazonia toquian Petrak is known on Ternstroemia toquian from Philippines (Hansford 1961). However, the new species differs from it in having mycelial setae. It is also similar to Meliola camellicola Yamam., but differs from it in having longer appressoria and mycelial setae.

Etymology: Named after its host plant.

\section{Mitteriella ziziphina Sydow}

Materials examined: TBGT 6432 PBL 25, 15.xii.2012, on leaves of Ziziphus sp. (Rhamnaceae), Nabagram, Kalpong, Diglipur, North Andaman, coll. V.B. Hosagoudar et al.

Rehmidothis osbeckiae (Berk. \& Br.) Theiss.

Materials examined: TBGT 6502 PBL 95, 8.xii.2012, on leaves of Melastoma malabathricum (Melastomaceae), Wrighte myo, riverside, South Andaman, coll. V.B. Hosagoudar et al.

\section{Schiffnerula azadirachtae Hosag. \& Sabeena}

Materials examined: TBGT 6459 PBL 52, 20.xii.2012, on leaves of Azadiracta indica (Meliaceae), Farrar gunj, South Andaman, coll. V.B. Hosagoudar et al.

\section{Schiffnerula chukrasiae sp. nov.}

(Fig. 18) urn:Isid:indexfungorum.org:names: 807706

Materials examined: TBGT 6681a (holotype), PBL 275a (isotype), 18.xii.2012, on leaves of Chukrasia sp. (Meliaceae), Central Nursery, Lorogic Forest Camp, Middle Andaman, V.B. Hosagoudar et al.

Colonies epiphyllous, thin to dense, up to $2 \mathrm{~mm}$ in diameter, confluent. Hyphae straight to flexuous, branching opposite, alternate to unilateral at acute to wide angles, loosely to closely reticulate, cells 17-27x5$6 \mu \mathrm{m}$. Appressoria alternate, unilateral, globose, entire, 17-10x8-10 $\mu \mathrm{m}$. Conidia of Questieriella scattered in the colonies, fusiform, straight to curved, slightly brown, 3-septate, constricted at the septum, end cells smaller and obtuse at the tip, 55-70x8-10 $\mu \mathrm{m}$. Conidiophores

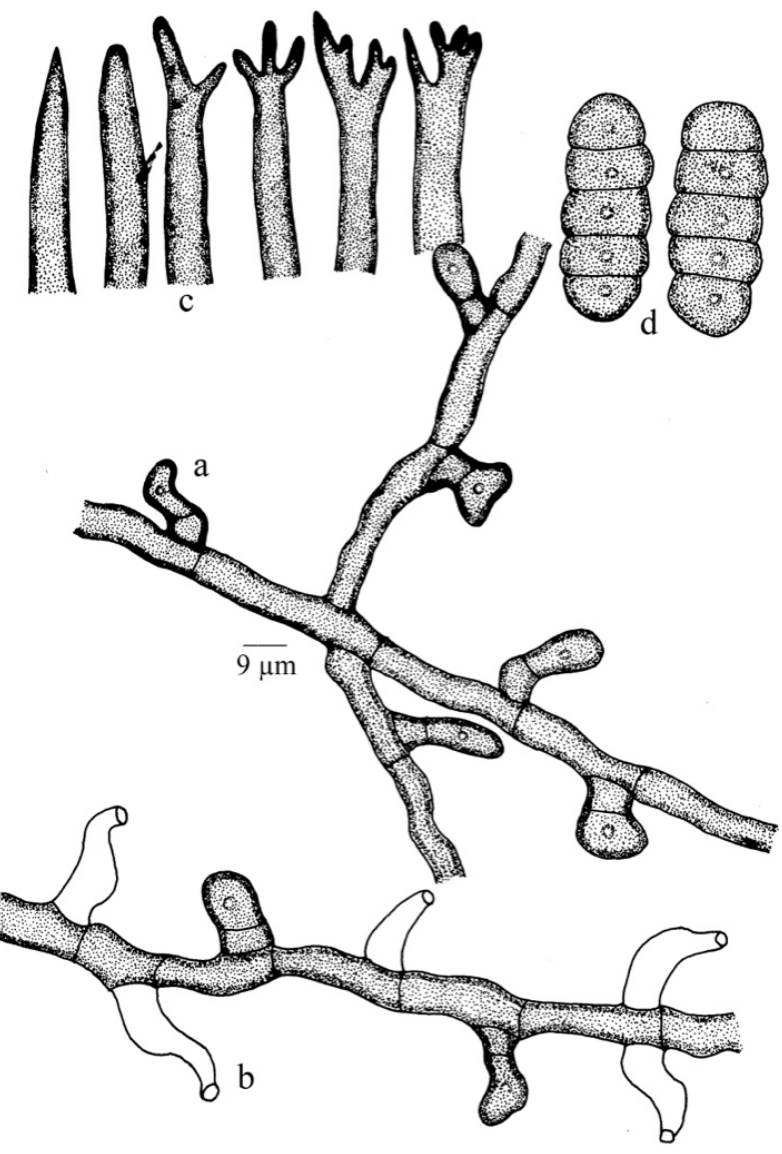

Figure 17. Meliola ternstroemiicola sp. nov.

a - Appressorium, b - Phialide, c - Apical portion of the mycelial setae; d - Ascospores 
of Sarcinella produced lateral to the hyphae, simple, straight to flexuous, macronematous, mononematous, 0-3 septate, integrated, cylindrical; conidia blastic, terminal, mostly sessile, solitary, dry, ovate to globose, sarciniform, brown to black, 4-7 celled, constricted at the septa, 20-27×22-30 $\mu \mathrm{m}$ in diameter. Thyriothecia scattered, orbicular, margin crenate to fimbriate, up to $80 \mu \mathrm{m}$ in diameter; peridial cells initially radiating, later central portion dissolved by exposing asci; asci few to many, globose, octosporous, bitunicate, up to $80 \mu \mathrm{m}$ in diameter; ascospores oblong, conglobate, uniseptate, constricted at the septum, 22-25 x 10-12 $\mu \mathrm{m}$, wall smooth.

Schiffnerulla entandrophragmatis Hansf. and Schiffnerulla azadirachtae Hosag. \& Sabeena are known on Entandrophragma anglolense and Azadirachta indica from Uganda, Nepal and India, respectively. Based on the host specificity, this fungus has been accommodated in a new species.

Etymology: Named after its host plant.

\section{Schiffnerula girijae Hosag. \& Archana}

Materials examined: TBGT 6473 PBL 066, 14.xii.2012, on leaves of Aegle marmelos (Rutaceae), Shipur, Diglipur, North Andaman, coll. V.B. Hosagoudar et al.; TBGT 6488 PBL 081, 20.xii.2012, Farrar gunj, South Andaman, coll. V.B. Hosagoudar et al.; TBGT 6518 PBL 111, 14.xii.2012, Radhanagar, Diglipur, North Andaman, coll. V.B. Hosagoudar et al.

Aegle marmelos is being cultivated in and around the temples and the teleomorph of the fungus is based on the few thyriothecia. However, it was surprising to locate this plant in the natural forest with very much robust leaves in contrast to tender leaves in the main land. Here, the fungus persisted mainly in teleomorph with few Sarcinella conidia.

\section{REFERENCES}

Farr, M.L. (1987). Amazonian foliicolous fungi - IV. Some new and critical taxa in ascomycotina and associated anamorph. Mycologia 79(1): 97-116.

Hansford, C.G. (1961). The Meliolineae. A Monograph. Sydowia 1-806pp.

Hansford, C.G. \& M.J. Thirumalachar (1948). Fungi of south India. Farlowia 3: 285-314.

Hosagoudar, V.B. (2008). Meliolales of India - Vol. II. Botanical Survey of India, Calcutta, 390pp

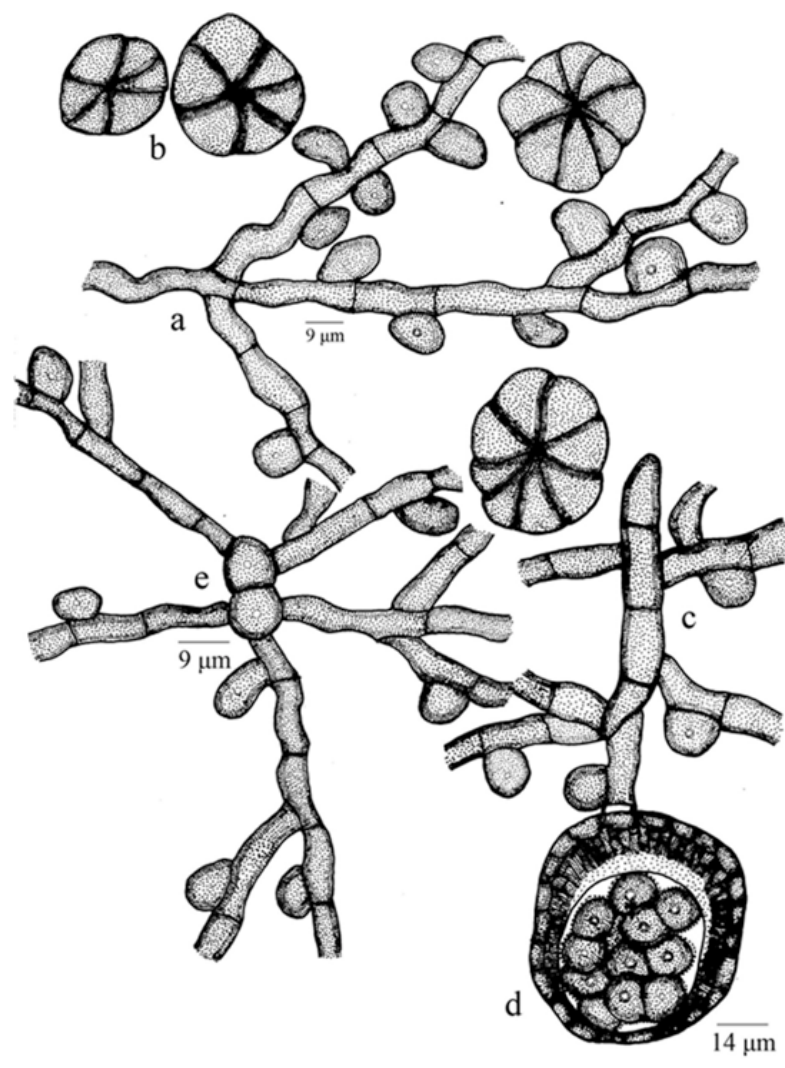

Figure 18. Schiffnerula chukrasiae sp. nov.

a - Appressoriate mycelium; b - Conidia of Sarcinella; c - Germinating conidia of Questieriella; $\mathrm{d}$ - Thyriothecium; e - Colony forming ascospore

Hosagoudar, V.B. \& D.K. Agarwal (2008). Taxonomic Studies of Meliolales. Identification Manual. International Book Distributors, Dehra Dun, 263pp.

Hosagoudar, V.B. (1996). Meliolales of India. Botanical Survey of India, Calcutta, 363pp.

Hosagoudar, V.B. (2009). The genus Asterina and its anamorph on Elaeocarpus species in southern Western Ghats in peninsular India. Journal of Applied and Natural Sciences 1: 27-30.

Hosagoudar, V.B. (2012). Asterinales of India. Mycosphere 2(5): 617852.

Hosagoudar, V.B. (2013). Meliolales of India - Vol. III. Journal of Threatened Taxa 5(6): 3993-4068; http://dx.doi.org/10.11609/ JoTT.03307.3993-4068.

Hosagoudar, V.B. \& T.K. Abraham (2000). A list of Asterina Lev. species based on the literature. Journal of Economic and Taxonomic Botany 24: 557-587.

Hosagoudar, V.B. \& S.P. Mathew (2000). A preliminary report on the mycoflora of the Andaman Islands, India. Journal of Economic and Taxonomic Botany 24: 631-640.

Hosagoudar, V.B. \& P.J. Robin (2011). Five new black mildews from the Western Ghats of peninsular India. Bioscience Discovery 02(2): $264-267$.

Hu, Y., Y. Ouyang, B. Song \& G. Jiang (1996). Flora Fungorum Sinicorum Volume 4 - Meliolales (1). Science press Beijing, 270pp+IVpls.

Hu, Y., B. Song, Y. Ouyang \& G. Jiang (1999). Flora Fungorum Sinicorum Volume 2 - Meliolales (2). Science press Beijing, 252pp. 